\section{EMBRYRIDDLE}

Aeronautical University

SCHOLARLY COMMONS
Journal of Aviation/Aerospace

Education \& Research

Volume 30

Number 1 JAAER 2021

Article 3

2021

\title{
Detectability of Clothing Color by Small Unmanned Aircraft Systems for Search and Rescue Operations
}

Ryan Blackburn

Embry-Riddle Aeronautical University, rlblackburn13@gmail.com

Robert Joslin

Embry-Riddle Aeronautical University, joslinr@erau.edu

Follow this and additional works at: https://commons.erau.edu/jaaer

Part of the Aviation Safety and Security Commons

\section{Scholarly Commons Citation}

Blackburn, R., \& Joslin, R. (2021). Detectability of Clothing Color by Small Unmanned Aircraft Systems for Search and Rescue Operations. Journal of Aviation/Aerospace Education \& Research, 30(1).

https://doi.org/10.15394/jaaer.2021.1886

This Article is brought to you for free and open access by the Journals at Scholarly Commons. It has been accepted for inclusion in Journal of Aviation/Aerospace Education \& Research by an authorized administrator of Scholarly Commons. For more information, please contact commons@erau.edu. 
Search and rescue (SAR) operations are conducted across the country in every climate and topography imaginable, many of them in remote environments with little to no access to public safety services (Goodrich et al., 2008). The United States Coast Guard (USCG) and the National Park Service (NPS) are the two primary federal agencies in the United States that conduct SAR missions (Heggie \& Amundson, 2009). In 2017, the most recent year that data was available, the USCG conducted SAR missions for 5,262 people, recovering 4,188 people alive. These missions included almost 17,000 sorties made by boats, manned aircraft, and cutters (United States Coast Guard, 2019). The NPS reported 4,194 SAR incidents in 2017. The total number of manhours expended on SAR operations by the NPS in 2017 exceeded 84,000 hours (National Park Service, 2020).

Search efforts put members of the search team into harm's way, potentially exposing them to the same hazards faced by the missing or injured person (Adams et al., 2007). Traditional aerial search and rescue methods generally involve the use of manned helicopters (Grissom, Thomas, \& James, 2006). According to data from the National Transportation Safety Board (NTSB), SAR helicopter accidents resulted in 32 fatalities between 1980 and 2013, with $43 \%$ of all SAR helicopter accidents resulting in fatal injuries. During the same period, only 19\% of general aviation helicopter accidents resulted in fatalities (Worley, 2015).

Search-and-rescue (SAR) operations are adopting small unmanned aircraft system (sUAS) technology to aid traditional human search parties operating on foot or by vehicle, which can be hindered by challenging terrain and obstacles; a limitation that sUAS can overcome by operating in the airspace above the ground search areas. Expediting the search process is critical, as the longer a person is missing, the greater the chance that they may succumb to any injuries or environmental hazards in the area. Unmanned aircraft can also be operated at lower altitudes and 
over more confined terrain compared to manned helicopters and airplanes that are also traditionally used to aid in SAR operations (Christie, Gilbert, Brown, Hatfield, \& Hanson, 2016). A potential solution for expediting the SAR search process is through aerial photography and image analysis software to detect the color of clothing worn by the person(s) being rescued. Many outdoor clothing manufacturers and public safety agencies advertise or recommend certain colors of clothing that are deemed as highly visible to the human eye, which may not be readily distinguishable by an optical sensor on a small unmanned aircraft (Heid, 2010; International Hunter Education Association [IHEA], n.d.; 3M, 2016). Furthermore, some states have enacted laws to require the use of these colors for outdoor activities, such as hunting. According to data collected by the IHEA, there are 43 states that require the use of hunter orange garments during at least some hunting seasons. The other seven states recommend the use of hunter orange garments. There are also a few states that allow new additional colors, such as hunter pink, but the majority still require hunter orange only. Consequently, it is critical to determine if traditionally accepted color choices are still the best option when considering aerial search operations utilizing sUAS. Previous studies on the use of sUAS for SAR have focused on thermal imagery, WiFi signals, or rudimentary image analysis of a few colors with shapes not representative of a human, rather than the use of automated software to detect a wide variety of different colors of clothing as worn by a person (Agcayazi, Cawi, Jurgenson, Ghassemi, \& Cook, 2016; Cooper \& Goodrich, 2008; Dinh et al., 2019; Eyerman, Crispino, Zamarro, \& Durscher, 2018; Goodrich et al., 2008; Khan, 2015; Sun, Li, Jiang, \& Wen, 2016; Van Tilburg, 2017). Weldon and Hupy (2020) discussed the limitations of using the traditional image analysis method of having individuals screen each image for potential matches, which is time consuming, leads to eye strain, and generally produces many false positive and missed detections. They 
proposed using Loc $8^{\mathrm{TM}}$ software to conduct the imagery analysis instead of using human analysts. Their research helped demonstrate the ability of Loc $8^{\mathrm{TM}}$ to conduct aerial image analysis; however, it did not look at how additional colors may affect the software's ability to make correct detections (Weldon \& Hupy, 2020). Determining clothing colors that are most detectable for sUAS SAR operations will inform individuals to include that color in their clothing and equipment and increase their chances of being found expeditiously in the event of an accident or getting lost.

\section{Research Hypothesis}

This research was designed to determine if a specific clothing color or collection of clothing colors make individuals more visible to sUAS optical sensor platforms when conducting SAR operations using automated software analysis of aerial imagery. The null hypothesis $\left(\mathrm{H}_{0}\right)$ was that clothing color will not have any effect on the ability to detect a missing person using image analysis software. The alternative hypothesis $\left(\mathrm{H}_{\mathrm{a}}\right)$ was that clothing color will influence the ability to detect a missing person using image analysis software.

\section{Literature Review}

As sUAS technology continues to improve, more and more researchers are looking for ways to incorporate the technology into public safety missions, such as SAR. Many different proposals have been presented, all of which have shown that sUAS SAR operations are not necessarily a new way of conducting business but are an additional tool that may be able to aid search teams.

\section{Aerial Search and Rescue}

Croft, Pittman, and Scialfa (2007) conducted a study on the ability of searchers to scan for notional aircraft crash sites from an aerial perspective. Testing was first conducted using 
aerial images of terrain with artificially imposed targets. In addition to the aerial images, searchers were flown over short routes where blue and white plastic sheets were placed to simulate the wing of a Cessna airplane. The objects being searched for were sufficiently large enough to be seen from the altitude of the flights. The intention of the research was to study gaze patterns of searchers and the sample size was relatively small $(\mathrm{N}=10)$. The findings indicated that the searchers were only able to identify the correct target $30 \%$ of the time (Croft et al., 2007).

Robinson (2012) noted that while conducting traditional manned aviation searches, airplanes cannot stop moving, allowing searchers only a short amount of time to search each area before the aircraft has moved on. To a lesser extent, the same is true of helicopters; while they can hover over an area to provide an extended search time, there are limitations on safe hover heights when out-of-ground effect. One of the benefits of capturing aerial images during these searches is that they can be studied for as long as necessary, by as many people as necessary, to ensure that there are no clues or items of interest in the image. Aerial images obtained from sUAS can also be significantly less expensive to obtain when compared to the cost of flying manned search missions (Robinson, 2012).

\section{Unmanned Aircraft Systems}

Van Tilburg (2017) reported on the first two known cases of sUAS platforms being used in SAR operations to capture and analyze images. The first was a case in Oregon where a rock climber had fallen. Ground search teams knew the approximate location of the individual from eyewitness accounts but did not have sufficient equipment to reach him. The equipment was ordered but would not be available until later that night. Having no knowledge of the injuries or state of the fallen climber, rescue teams had no choice but to prepare to conduct a dangerous 
nighttime climb to locate the victim in a slot canyon. An observer in the area offered to use their personal sUAS to attempt to ascertain the fate of the climber. The SAR team, which was on standby until the new equipment arrived, allowed it. The sUAS operator was able to locate the victim and return footage to the SAR team notifying them that the victim was deceased. The use of the sUAS prevented the SAR team from making the dangerous climb at night and allowed them to schedule a recovery operation the following day when it was safer. The second case was an sUAS used to search difficult to reach areas to prevent ground teams from having to expend time in those areas. On the second day of search, an sUAS with a thermal camera was used to capture images of a creek bottom that was difficult to see from the creek banks. Five total flights were conducted on the second search day and eliminated $2.7 \mathrm{~km}$ (1.7 miles) that ground teams did not need to search. On the third search day, another sUAS was used to clear cliffs and the surrounding scree fields. The cliffs were difficult to clear from the ground. Rappel teams were used to clear one section of the cliff face that the sUA (small unmanned aircraft) could not access, but all other areas of the cliff face were cleared by the sUA (Van Tilburg, 2017).

Drone manufacturer DJI has reported a total of 444 people saved through the use of sUAS at the time of this paper. An interactive map created on their website shows the countries and SAR events. Search and rescue operations involving sUAS have been reported in 29 different countries, with the majority being in North America, followed by Europe. Of the North American SAR events, less than 10 took place in Canada, with the rest in the United States. The DJI statistics and provided map do not include instances where an sUAS was used to aid in the search but was not a contributing factor in the rescue. For example, if the SAR team employs an sUAS, but the missing person is found by the ground team without the aid of information from the sUAS, the rescue is not included on the map or in the reported rescues. Even though the data 
was compiled by DJI there were no limitations on which sUAS was used in the search, or who the manufacturer was (DJI, n.d.).

Cooper and Goodrich (2008) researched the limitations of various display layout configurations for sUA operators and sensor operators. The researchers first investigated using two screens, one for the operator which showed telemetry, command, and control data, and one for the sensor operator, which showed the live feed video. They found that the sensor operator was generally not able to determine the location or flight path of the sUA unless they were near the operator and could see both display screens. The researchers had participants watch a simulated sUA flight on a control display that also showed a live feed from the sUA camera. The participants were directed to mark on the screen several target spheres as they found them in the live feed. The researchers found that the participants were not able to determine which objects they had previously marked, as they could not keep track of both the live feed video and the flight path of the sUA. While there were only 10 target spheres in the simulated flight, the participants marked an average of 16.35 targets. The researchers suggested that it would be possible for a single operator to control the sUA, observe the video feed, and detect the targets, only if the targets are sufficiently easy to detect (Cooper \& Goodrich, 2008).

Dinh et al. (2019) proposed using sUAS platforms to scan for mobile device signals when attempting to locate missing persons. The researchers suggested that sUAS could be used to detect an SOS signal from a mobile device, even if the missing person was not located in an area with cellular reception. This would be possible through the use of an application that must be previously downloaded onto the device, when service allows, that would then send out an emergency signal when needed. The sUAS flying over the device would detect the signal and could then use it to triangulate the location of the missing person. Unfortunately, this solution 
requires a missing person to have the foresight to download the required application, have sufficient battery life to broadcast the signal, and be conscious in order to enable the application when an emergency arose (Dinh et al., 2019).

Eyerman et al. (2018) released a study detailing joint research into sUAS SAR operations conducted by sUAS manufacturer DJI, the European Emergency Number Association (EENA), and research firm Black Channel. Initial testing was conducted in 2014 by Black Channel, who found that sUAS had the potential to aid in SAR operations, but that the technology in 2014 was not where it needed to be in order to provide any value in search operations. After allowing the industry to mature for a few more years, the three companies went back and conducted more testing. The follow-on study found that, when equipped with more modern sUAS platforms, the search teams were able to locate the search subject approximately three minutes faster than teams that were not equipped with an sUAS. However, the study also found that sUAS-equipped teams were not able to locate the search subject as often as the non-equipped teams. One of the reasons the researchers determined that the sUAS were less effective was that the target was a black and yellow swift water rescue suit and the suit color made it difficult for sUAS operators to notice during sunny weather. The researchers noted that red showed up better in the sUAS video; however, they did not conduct the study using a different colored target object. The searches in this study were based on sUAS operators searching in areas determined by the SAR team and observing the live feed to attempt to locate the search subject while also controlling the flight path of the sUA (Eyerman et al., 2018).

Goodrich et al. (2008) discussed using sUAS in wilderness SAR. In their study, they found that one of the limitations was the quality of video available from the sUAS and the ability to accurately define what area a specific image covers. Since this study was conducted, more 
and more sUAS platforms are equipped with Global Positioning System (GPS) receivers, which can completely eliminate the problem of image location by geotagging each image with coordinates automatically. One of the suggestions that the researchers made was that the use of automated detection programs could significantly aid the search party, as they would not need to rely on either the pilot or sensor operators, who have other duties during flight in addition to searching. Alternatively, they also suggested that post flight evaluation of images would reduce operator load during the mission (Goodrich et al., 2008).

\section{Automated Software and Algorithms}

Khan (2015) explored using computer algorithms to perform automated person detection using thermal imagery obtained from an sUAS platform. This research was limited due to the use of a Raspberry Pi based computer that was not able to handle higher level processing algorithms. The result was the use of a less powerful algorithm that had mixed results. Additionally, thermal technology for sUAS has seen more limited adoption than sUAS platforms themselves, likely due to the high costs and low resolutions available when compared with conventional optical cameras. The thermal sensor used by Khan required that the unmanned aircraft be flown at lower altitudes in order to make a successful detection. Past 40 meters (131 feet), the thermal sensor was not able to capture a clear enough image for the algorithm to detect a human shape (Khan, 2015).

Al-Kaff, Gómez-Silva, Moreno, Escalera, and Armingol (2019) designed a system that would filter flagged images by running them through a human pose filter. The filter would reduce the number of false positive detections when searching for human subjects by analyzing images and only selecting items with a similar shape to the human body. The software detects the location of the head, and then measures the angles and length of attached body parts to ensure 
that it has roughly human dimensions. If the object passes the filter, it is flagged as human; otherwise it is rejected. Throughout the paper, many images are included of human subjects lying in various poses and being detected by the software. However, there does not seem to be any data supporting the software's ability to detect a standing person in an image captured from directly overhead, or of a human subject not in a supine position. Additionally, based on the explanation of the detection algorithm, a person whose body is mostly or partially obscured would likely be rejected as a false identification. The researchers noted that the use of this software resulted in no false positive results; however, it is unclear how many missed detections it would have in a larger study with more variation to the positioning of the human subjects (AlKaff et al., 2019).

Morse, Thornton, and Goodrich (2012) developed a method of evaluating pixel colors in aerial images and determining if the image contained pixels of contrasting colors. For example, a blue shirt would be a significant contrast to a desert environment consisting only of sand dunes. The software would then alert the operator of the detection and suggest it as a possible target. The researchers looked at 24 target items in aerial video, with half being suggested as possible targets by the software, and the other half not being suggested. The researchers found that detection rates were higher when using target suggestions from the software, allowing searchers to identify objects that they may not have seen without the aid of the software. Since this detection method only looks at contrasting colors, it will not miss an object because it does not have the correct shape, as some other methods will. However, this method is only useful when searching for objects that are contrasting to the background. If a missing person were lost in a grassy area and wearing green, it is unlikely that the software could detect them. However, the search method described only uses the software as an additional tool, allowing the individual 
searchers to still look through images for non-flagged items, potentially allowing detection of an unflagged item, just at a lower detection rate (Morse et al., 2012).

Sun et al. (2016) used a Talon sUAS from X-UAV. The Talon is not a ready-to-fly model, as it requires the purchase and installation of additional components and at least some knowledge of sUAS design and construction. This makes the Talon an unlikely choice for most SAR teams and public agencies. The researchers designed an on-board algorithm to detect variations in the color of target objects for identification of people, vehicles, or even crashed aircraft. The camera was modified to record in YUV color space, as opposed to the more common RGB color space. The YUV color space is more commonly used in television systems. It uses a luminance signal $(\mathrm{Y})$, known as luma, and two color signals ( $\mathrm{U}$ and $\mathrm{V})$ known as B-Y (blue minus luma) and R-Y (red minus luma). For television, the YUV signal is converted to RGB by the display system (Wang et al., 2017; Hunold, 1999). The algorithm would then compare the red and blue color variances for all of the pixels and look for pixels that were in high contrast to the rest of the pixels. This pixel variance analysis allowed the software to identify anomalies and flag them for user review. One of the limitations of this type of analysis is that it cannot detect people or objects that are similar in color to their surroundings. The systems also had no way to filter out flagged images that were not items of interest, which resulted in many false positives, as there was no method to narrow down the search (Sun et al., 2016).

Marshall and Perkins (2015) investigated the ability of various algorithms to detect colors that were anomalies compared to surrounding colors. Six different terrain types were used in this evaluation; however, no aerial images were captured for this research. The researchers instead used extant images that they selected and superimposed target images onto them. The target 
images were reduced in size and blended slightly to give the appearance that they were originally in the image. The terrain images were evaluated for detection ability and processing times across eight algorithms. Additionally, the images were converted into different color spaces prior to analysis to determine if certain color space and algorithm combinations would produce better results (faster or more accurate) (Marshall \& Perkins, 2015).

Hoai and Phuong (2017) examined the ability of imagery analysis software to detect color anomalies in aerial images. This study was focused on finding the algorithm and color space combination that proved to be the most successful in detecting an anomaly. Eight different color space options were analyzed with three different terrain types and eight different analysis algorithms. The researchers found that different combinations of algorithm and color space were successful in different terrain types. There were several limitations to this study. The aerial images analyzed were captured from 600 meters (1,968 feet), which is significantly higher than most sUA will be flying during SAR operations. The higher altitude images would reduce the sharpness of terrain features when compared to an image from the same camera at a lower altitude. Additionally, the images analyzed were not originally captured for the purpose of this research. The images were repurposed from another study, and target items were superimposed into the images after capture, which would give the target a different pixel structure and coloration than if it was captured in the original image (Hoai \& Phuong, 2017).

Agcayazi et al. (2016) designed a semi-autonomous sUAS that conducted its own flight planning in addition to anomaly detection. The researchers began with a human detection algorithm that was abandoned early in the project due to its inability to detect objects that were not in specific positions. The anomaly detection process they selected next was similar to those previously discussed, relying on detecting objects of interest based on differences in color 
compared to surrounding pixels. The algorithm identifies the section of the image with the biggest anomaly and highlights it for the operator to review. This method of highlighting only the largest anomaly would potentially allow a person to go undetected in an image if there were another, slightly larger object in the same image that was different in coloration than its surroundings (Agcayazi et al., 2016).

\section{Loc8 $^{\text {TM }}$ Image Analysis Software}

Loc $8^{\mathrm{TM}}$ was designed for use with sUAS platforms to conduct SAR operations in any area, even without Internet access. The program works by scanning individual images for user defined colors at the pixel level. Once a pixel (or cluster of pixels, depending on setup) is identified as matching the search parameters, the image and matching pixels are flagged for user review. Loc8 ${ }^{\mathrm{TM}}$ is designed to work with all sUAS and optical camera platforms (Loc8, n.d.-a). Antunes (2020) reviewed the Loc $8^{\mathrm{TM}}$ software in a descriptive article that explained many of the capabilities and benefits of the image analysis software. One of the co-founders of the Loc8 ${ }^{\mathrm{TM}}$ company noted that many times operators focus on the hardware side of sUAS, ensuring they have the newest equipment, and fail to focus on the software side, which can be equally as important. According to one of the software's designers, it can take up to two minutes for a trained image analyst to search a 20-megapixel image. At a rate of two minutes per image, 100 images would take over three hours to scan by a single individual, and most sUAS SAR operations produce hundreds, if not thousands, of images that need to be analyzed. The Loc $8^{\mathrm{TM}}$ program can scan 100 20-megapixel images in less than five minutes (Antunes, 2020).

Weldon and Hupy (2020) conducted research using Loc $8^{\mathrm{TM}}$ software to perform the imagery analysis instead of using human analysts. The sUAS used by the research team was a specialized platform, the C-Astral Bramor PPX, that the researchers admitted was likely outside 
the budget and skillset of first responder teams. The researchers note that there are less complex platforms that can be used to conduct searches with somewhat similar results; however, none of the ones currently available have the advanced capabilities used in this study. The research in this study was designed to look at the ability of the Loc $8^{\mathrm{TM}}$ software to detect a missing person while conducting a full aerial search of an area. Seven items were placed in a field in locations unknown to the Bramor PPX operator. The operator commanded the aircraft to fly a lawnmower-style flight pattern over the search area, and the aerial images collected were loaded into Loc $8^{\mathrm{TM}}$ for analysis. This research did not consider the effect the color of the search objects might have on the Loc $8^{\mathrm{TM}}$ software. The colors of the items that the team searched for included one off-white shirt, two black shirts, one pair of blue jeans, one light blue shirt, one dark blue shirt, and one skeleton with blue jeans and a checkered white and black shirt. This means that even though seven search items were used, they only fell into three color groups (blue, black, and white) without any additional variation in color. The team did note that one color was too similar to the background (off-white shirt) and discarded it from future search considerations. This research helped demonstrate the ability of Loc $8^{\mathrm{TM}}$ to conduct aerial image analysis; however, it did not look at how additional colors may affect the software's ability to make correct detections (Weldon \& Hupy, 2020).

\section{Clothing Color}

The International Hunter Education Association (IHEA) continues to recommend that all hunters wear hunter orange (also referred to as blaze orange) clothing while in the field for visibility. The recommendation is based on safety, ensuring that other hunters will not mistake a person for an animal through foliage. According to data collected by the IHEA, there are 43 states that require the use of hunter orange garments during at least some hunting seasons. The 
other seven states recommend the use of hunter orange garments. There are also a few states that allow new additional colors, such as hunter pink, but the majority still require hunter orange only. The states allowing hunter pink in place of hunter orange appear to have arrived at the decision not based on any additional scientific study but in an effort to attract more female hunters. Additionally, six of the 10 Canadian provinces require the use of hunter orange while hunting, and two of the others recommend its use (International Hunter Education Association [IHEA], n.d.).

The United States Forest Service also recommends wearing hunter orange, even by nonhunters visiting national forests during hunting seasons. For non-hunters, the Forest Service also recommends bright color clothing, such as red, orange, and green. Visitors should avoid white, black, brown, earth-toned greens, and animal colored clothing. There are also recommendations for dogs to wear orange vests if they accompany people into the forests (United States Forest Service, n.d.)

The 3M company (2016) published a regulatory update concerning the newest regulations for high visibility garments. The American National Standards Institute, Inc. (ANSI) has established regulations concerning the type and visibility of garments for certain industries for over two decades. Industries that are required to comply with ANSI standards include construction, utility, emergency responders, airport ramp personnel, and off-road workers. The ANSI standards, known as the American National Standard for High-Visibility Safety Apparel and Accessories, includes provisions for all types of clothing worn by workers in applicable industries to help make them more visible during the day, at night, and during periods of reduced visibility. All classes of ANSI high-visibility garments require at least some portion of retroreflective material, which reflects a high portion of light back at the source it originated 
from. The requirement for retroreflective material is unlikely to provide extra detection for sUAS SAR operations, as most unmanned aircraft are not equipped with directional lights that would be reflected. However, ANSI also requires that a large portion of the non-retroreflective material be colored fluorescent material that is highly conspicuous and contrasting to the surrounding environment (3M, 2016).

Zaidi et al. (2007) reported that the peak wavelength that humans are able to perceive is 555 nanometers. This corresponds to a color that is partway between green and yellow (Zaidi et al., 2007). While this is reportedly the color that the human eye is most sensitive to, it is not the color generally recommended by safety agencies. This is likely due to the lack of contrast between the color green and many areas that people generally find themselves. Green is a color that occurs often in nature, making the continued recommendation of oranges and yellows reasonable.

\section{Methodology}

This experimental research examined the ability of automated image analysis software $\left(\operatorname{Loc} 8^{\mathrm{TM}}\right)$ to identify various colors of clothing from aerial images captured by an sUAS. Data was collected utilizing three sUAS models with different sensor payloads. The first was the Mavic 2 Pro from Chinese sUAS manufacturer DJI. The Mavic 2 Pro is equipped with a gimballed Hasselblad 1 in. complementary metal-oxide semiconductor (CMOS) sensor which captures images of 20 megapixels. It has a field of view of approximately 77 degrees, a focal length of $10.26 \mathrm{~mm}$ and an adjustable aperture of f/2.8-f/11 (Alfio, Costantino, \& Pepe, 2020). The second sUAS was the Bebop 2 from French manufacturer Parrot. The Bebop 2 has a 14megapixel 1/2.3 in. CMOS camera (Suciu et al., 2018). The Bebop 2 camera has a nonadjustable aperture of f/2.3 and a focal length of approximately $2 \mathrm{~mm}$ (Pagliari \& Pinto, 2018). 
The field of view on the Bebop 2 is listed as 180 degrees, which is misleading. The Bebop 2 camera is not gimballed but supports camera pan and tilt through the use of software, which gives the appearance of camera movement, but in reality, is just the system using only sections of the sensor and lens (Goldman, 2016). The actual demonstrated field of view is approximately 80 degrees (Nicolas, 2105). Aerial images captured by the Bebop 2 are not rectilinear as the other two sUAS sensors but captured in a fish-eye format. The third sUAS was the Parrot Disco. The Disco is the only fixed-wing model sUAS used. The Disco comes equipped with the same camera used in the Bebop 2 sUAS (Boyles, 2017). In order to ensure that three different sensors were used for data collection, the Disco was modified to carry a non-gimballed GoPro Hero8 camera oriented for nadir image capture. The plans used to modify the Parrot Disco are available online (Skyvue 3D, 2017). The GoPro Hero8 Black edition offers 12-megapixel images with a focal length of 16-39mm, depending on the digital lens selection (GoPro, n.d.). It has a 1/2.3 in. CMOS sensor, 2x digital zoom, and a non-adjustable aperture of f/2.8 (Michael, 2020).

\section{Flight Planning Software Selection}

Flight planning for missions involving the DJI Mavic 2 Pro and the Parrot Bebop 2 were conducted using the Pix4D Capture application on either an iPhone $X$ or an iPad Mini 5. Pix4D allows the operator to plan a grid-style mission while controlling the aircraft speed and the side and front overlap of images captured. Once the flight plan is built, the Remote Pilot in Command (RPIC) commands the software to begin and the flight is conducted autonomously from that point (Fernández-Lozano et al., 2018). Pix4D Capture does not allow operators to set a discrete speed for flights. Operators chose between one of five speed settings for the flight, then the software will adjust the actual speed based on the length of the current flight leg and the wind 
conditions in which the sUA is operating. For both the Mavic 2 Pro and Bebop 2 missions the speed setting was set to the default of "Fast." The fast setting allows the aircraft to complete longer missions than lower settings due to the increased maximum flight speed available. Additionally, the fast setting ensures that the camera angle is the same for all images on each leg when not operating with a 90-degree camera angle (i.e. Bebop 2 missions) (Pix4D, 2018). Aerial images were monitored in real-time by the RPIC to ensure that the speed setting did not cause blurry image captures. The base-model Parrot Disco is not supported by the Pix4D Capture application (Pix4D, n.d.). The Parrot Disco was controlled via the FreeFlight Pro application from Parrot, with the internal Flight Plan function. The Flight Plan function allows the RPIC to create a series of waypoints for the Disco to follow as it completes its mission. There is not a grid-style flight plan option, so the flight plan must be manually created by the RPIC waypoint by waypoint. However, once the flight plan is created, it can be saved and reused for all subsequent flights in the same area, thereby enhancing the repeatability of the experiment (Parrot, 2019). The speed of the Disco cannot be altered when using the Flight Plan section of the FreeFlight Pro application.

\section{Torso Model Design}

Ten torso models were constructed using chicken wire mesh, each covered with a different garment color, as shown in Figure 1. The models had the same chest and waist dimensions as the Hybrid II Anthropometric Test Dummy (ATD) used by the Federal Aviation Administration (National Institute for Aviation Research, 2013). Eight non-enhanced visibility shirts and two enhanced visibility shirts were selected for this research project. The two enhanced visibility shirts were high visibility yellow and high visibility orange. The colors of the non-enhanced visibility shirts were selected to represent the primary and secondary colors on 
a standard color wheel: red, yellow, blue, orange, green, and purple. Additionally, one black shirt and one white shirt were used to supplement the color options. The eight non-enhanced visibility shirts were all made of cotton and the enhanced visibility garments were made of polyester.

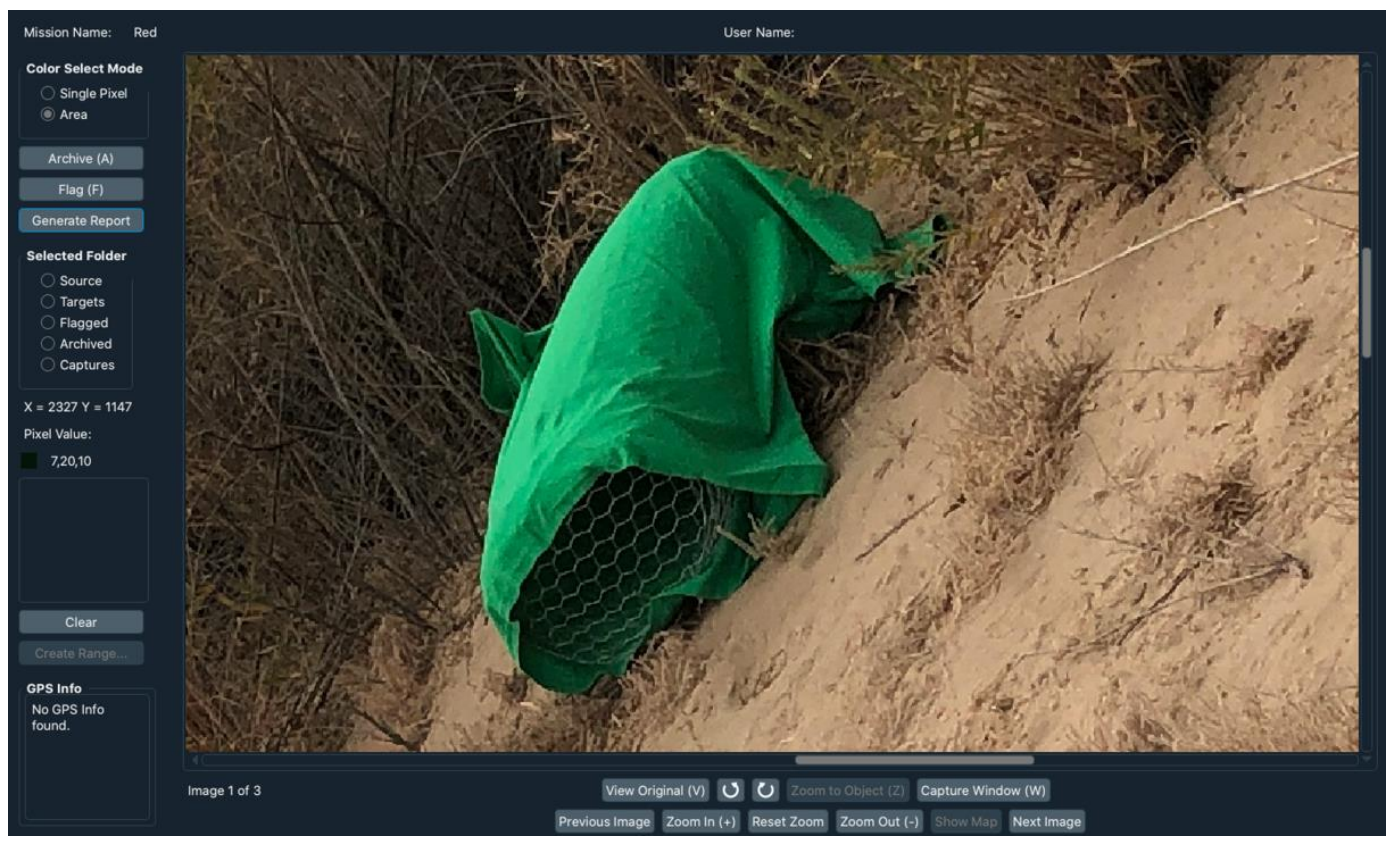

Figure 1. Green shirt on chicken wire torso.

\section{Flight Area Setup}

At each flight area, all 10 torso models were laid out in a supine position, representing a person lying down. The models were not placed in any particular order or design at the flight locations. All torsos were placed close enough so that each sUA optical sensor could capture all 10 models in a single image. At each area, the models were placed in similar conditions for an equal chance at detection (i.e. all models in direct sunlight or all models in shade). The stand-off slant range between the sUA and the torsos varied based on the altitude flown but ranged between 0 feet (sUA directly over torso taking nadir images) and approximately 500 feet at 400 feet AGL. Multiple flights were conducted within each area on different days, time of day, and 
shading to minimize the effects of any covariates or confounds. Prior to each block of flights, the RPIC checked weather data in both the Hover and UAV Forecast iOS mobile applications. In addition to the forecast, wind and temperature readings were taken by the RPIC with a handheld anemometer to ensure Visual Meteorological Conditions (VMC) and local measured wind speeds and temperatures were below sUA maximums.

\section{Data Collection}

The data used for this research consisted of aerial images captured by three different sUAS and sensor combinations in a rural/remote desert summer daytime environment at six locations with varying topography and vegetation in the vicinity of Yuma, Arizona. Flights were conducted at four altitudes (100 feet above ground level (AGL), 200 feet AGL, 300 feet AGL, and 400 feet AGL) during four time blocks to more effectively simulate SAR operations. Eighteen flights were conducted, with each flight covering all four altitudes with each altitude containing images of all 10 torso models, yielding 72 sets of data. All images were nadir except for those obtained from the Parrot Bebop 2. The Bebop 2 uses a fixed 30-degree downward camera angle that is non-adjustable with the flight planning software (Pagliari \& Pinto, 2018). All three sUAS gathered images at each flight area within one hour of each other during each time block (9 a.m., Noon, 3 p.m., 6 p.m.) to ensure similar sunlight and atmospheric conditions. Examples of an image capture at $100 \mathrm{ft}$ AGL for the DJI Mavic 2 Pro and the Parrot Bebop 2 are shown in Figure 2 and Figure 3. 


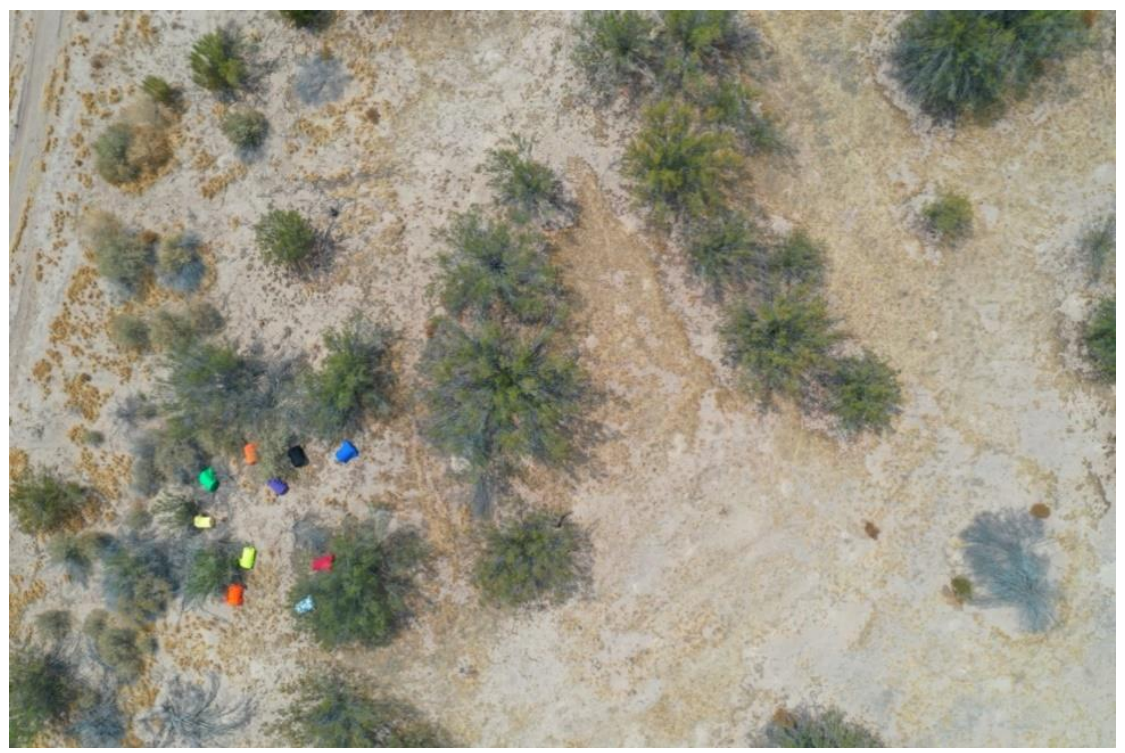

Figure 2. Example aerial image capture from the DJI Mavic 2 Pro at 100 feet AGL.

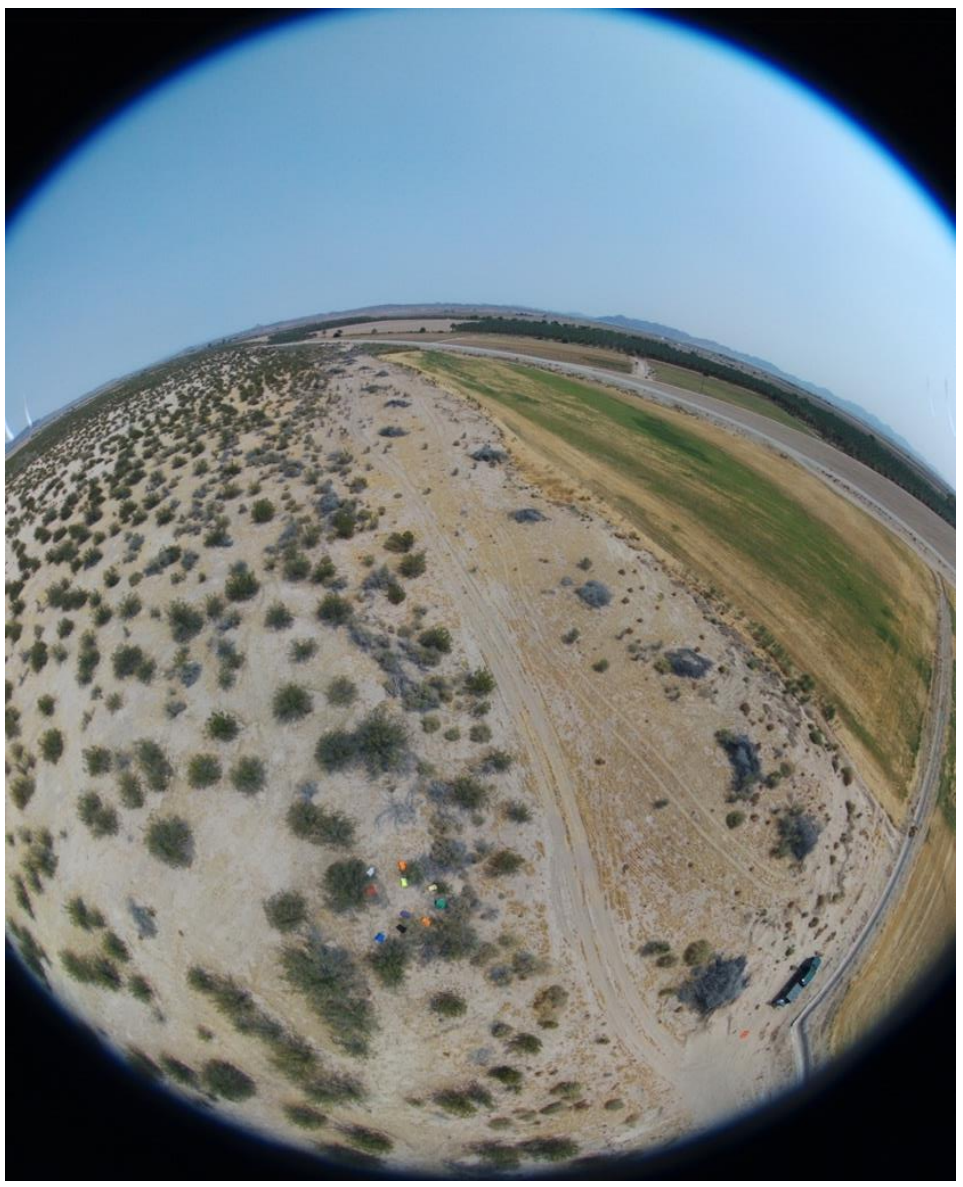

Figure 3. Example aerial image capture from the Parrot Bebop 2 at 100 feet AGL. 


\begin{abstract}
Analysis
Images from each of the three sUAS sensors were analyzed using the Loc $8^{\mathrm{TM}}$ program running on a 2020 MacBook Pro. The Loc8 ${ }^{\mathrm{TM}}$ license type for this research was Basic, and the Loc $8^{\mathrm{TM}}$ version number was 2.53 . Loc8 ${ }^{\mathrm{TM}}$ program works by scanning individual images for user defined colors at the pixel level. Once a pixel (or cluster of pixels, depending on setup) is identified as matching the search parameters, the image and matching pixels are flagged for user review. Color profiles were built within the Loc $8^{\mathrm{TM}}$ program prior to initial aerial image analysis using the Loc8 $8^{\mathrm{TM}}$ Bloodhound Technique. The Bloodhound Technique is the Loc8 ${ }^{\mathrm{TM}}$ recommended procedure for SAR operations (Loc8, n.d.-a, n.d.-b). The recommendation for this technique comes from previous SAR experience with traditional searches where teams are told what the missing person was last seen wearing. For sUAS SAR operations, the Bloodhound Technique is based off the last image available of the missing person. The image is used to define the color profile that Loc $8^{\mathrm{TM}}$ uses to analyze images.

\section{Loc8 $^{\mathrm{TM}}$ Color Palate Creation Process}

For this research, an image capture from an iPhone $\mathrm{X}$ was taken at ground level of all 10 garments for color palate creation, as shown in Figure 4. 


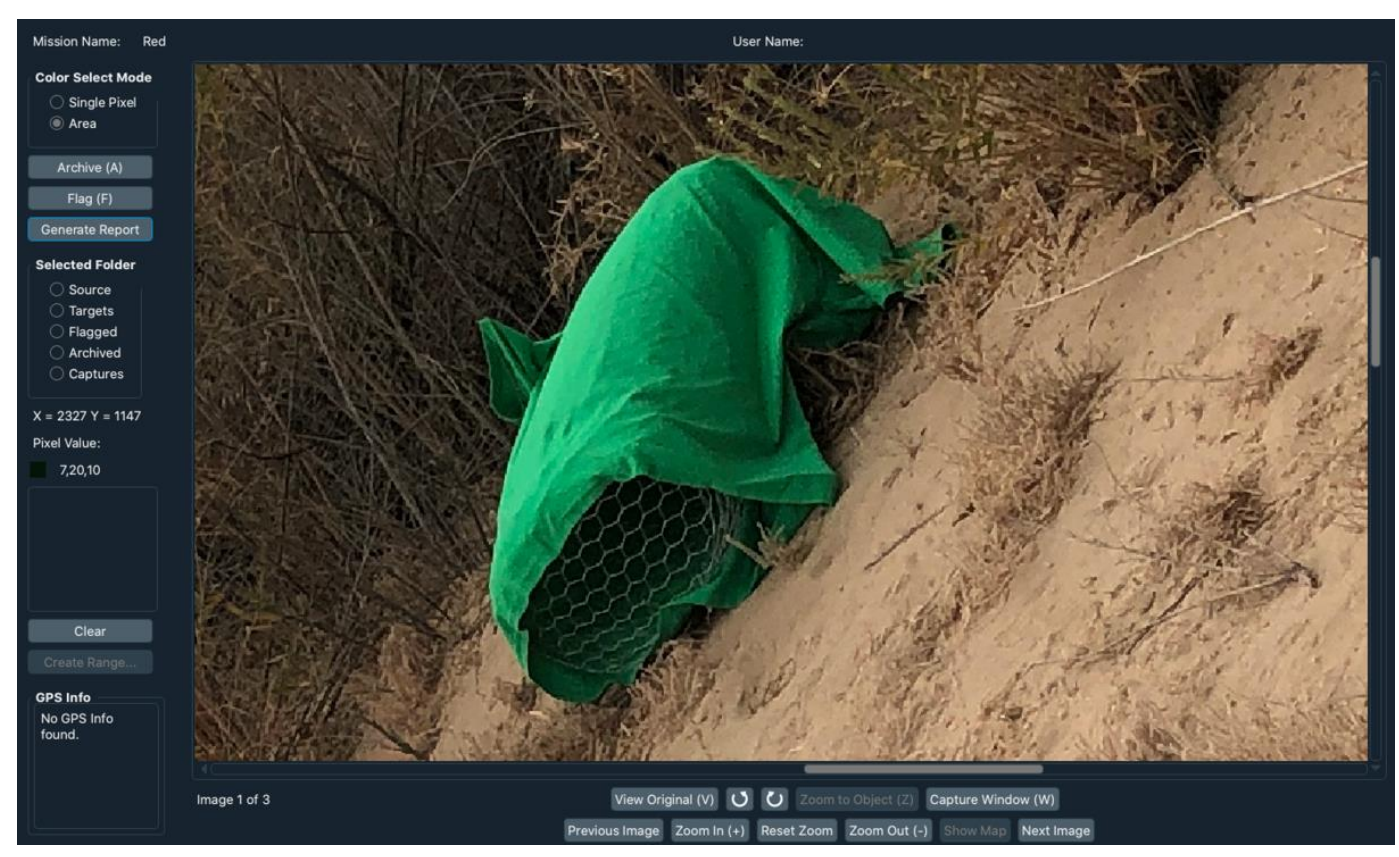

Figure 4. Step one begins with the Loc8 ${ }^{\mathrm{TM}}$ Viewer screen with the image being used to create the profile open. The operator then zooms into the image focusing on the color of interest until individual pixels can be seen in the image (Loc8, n.d.-b).

After uploading the image into the Loc $8^{\mathrm{TM}}$ viewer screen, the operator increases the zoom on the image until individual pixels can be viewed, as shown in Figure 5. The operator then selects either several individual pixels or a wide area of pixels. The selected pixel RGB values are shown on the left side of the screen. 


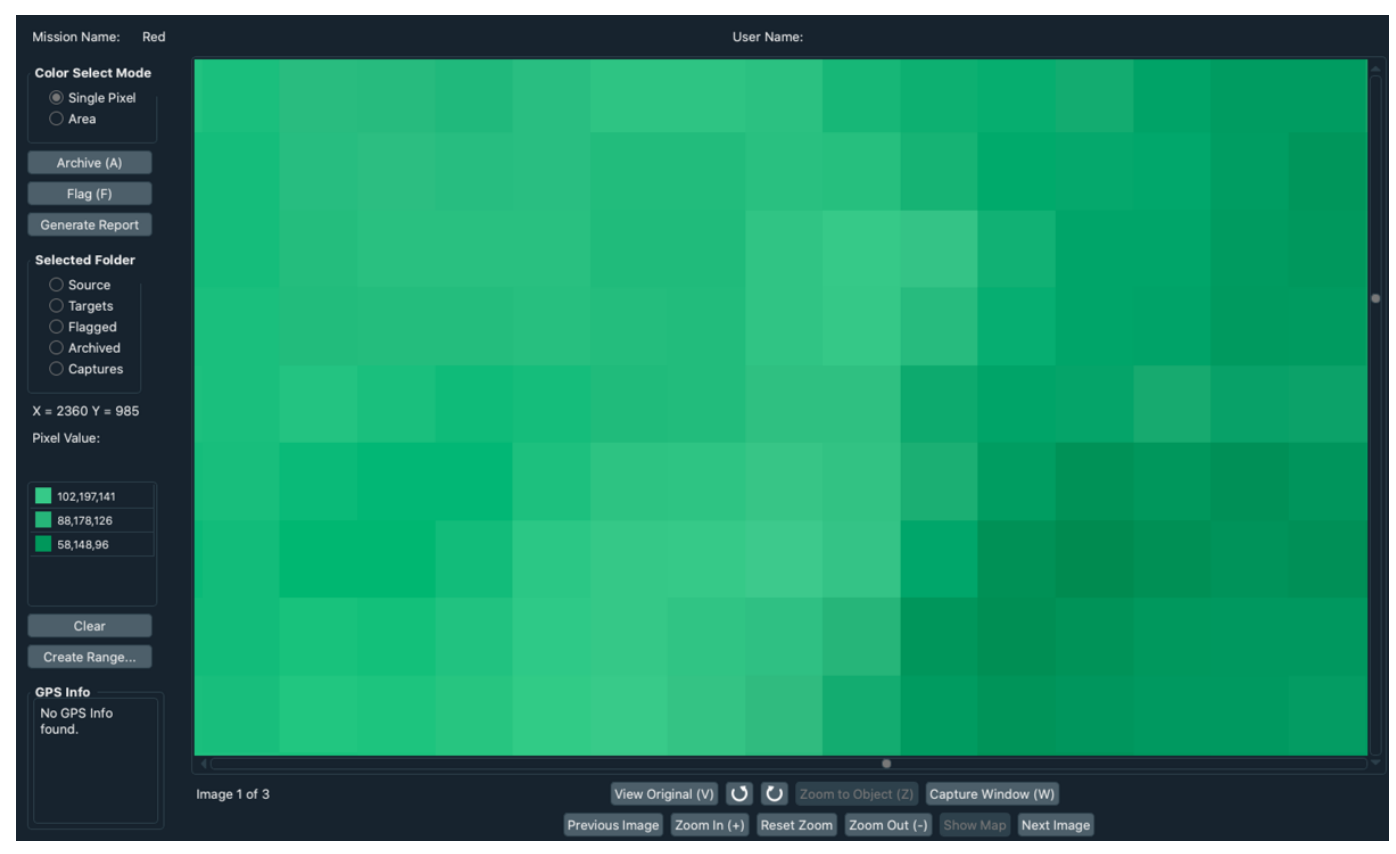

Figure 5. The operator can either select individual pixels from the image or select an area of pixels from the image. For this research, the individual pixel method was utilized. The three selected pixels are identified on the left section of the screen with their corresponding RGB values (Loc8, n.d.-b).

After selecting all desired pixels, the operator creates an average of all pixel values. The average is then converted into a range by Loc $8^{\mathrm{TM}}$. The default range size is 10 but can be increased or decreased as needed. Once the range is created, the color wheel shows a visual representation of the colors that Loc8 ${ }^{\mathrm{TM}}$ will search for when using the created range, as shown in Figure 6. The operator then saves the color palate and can load it into Loc8 $8^{\mathrm{TM}}$ as needed to conduct image analyses. 


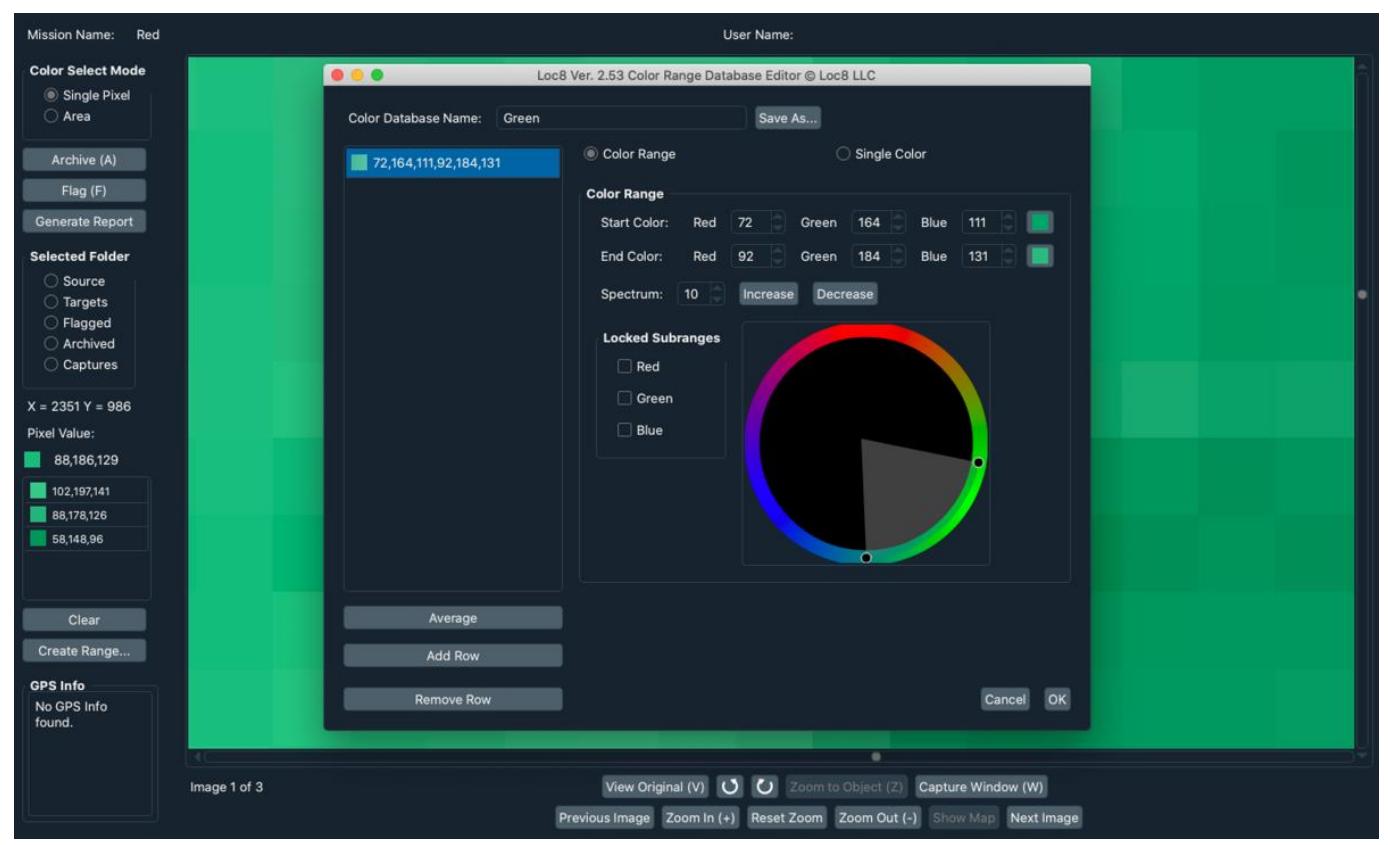

Figure 6. The selected pixels are averaged and then converted to a range by Loc8 ${ }^{\mathrm{TM}}$. The default setting when creating the range is 10 , which creates a color range that is 10 above and below each of the three RGB color values determined by averaging the previously selected pixels. The size of the range can be increased or decreased by the operator (Loc8, n.d.-b). For this research, the range was left at the default value.

\section{Statistics}

The torso models were placed in the same area, so that a single image capture could contain all 10 torso models. The images collected from each flight were analyzed using the prebuilt color profile for each color of clothing. The number of successful detections of each color were recorded in separate tallies based on the altitude of the sUA and the sensor that captured it. Every garment color had an equal number of chances for detection at each altitude. The same images were re-analyzed 10 total times, once for each color. The data collected from the Loc8 ${ }^{\mathrm{TM}}$ imagery analysis is dichotomous (i.e. detected vs. not detected). A chi-square test for independence was conducted to determine if detection rates across all colors were equal, and therefore accept or reject the null hypothesis. The chi-square test was chosen as the data collected was nominal and the chi-square test does not require homoscedasticity in the data. The independent variable was the color of the clothing, which had 10 levels based on the number of 
colors (red, yellow, blue, orange, green, purple, white, black, high visibility yellow, and high visibility orange). The dependent variable was the number of successful detections that the Loc $8^{\mathrm{TM}}$ software returned after analyzing the sUAS imagery. The dependent variable had two levels: detected and not detected. A Cramer's V was conducted to determine the strength of the test.

\section{Results}

Each sUA flight conducted collected a varying number of images, based on the sUA used and the altitude at which the flight took place. For the two quadcopter sUA (Bebop 2 and Mavic 2 Pro) the initial flight pattern was created in Pix4D Capture at a flight altitude of 100 feet AGL and saved. Subsequent flight patterns used the saved 100 feet AGL file with only the altitude parameter adjusted. At higher altitudes, it took each sUA less time to cover the same distance, resulting in shorter flights with less total image captures. This was less apparent on the Bebop 2, as the Pix4D Capture application required the flight path to be widened with each increase in altitude in order to maintain the same number of legs for the flight, resulting in similar numbers of total image captures at higher altitudes, as shown in Table 1.

Table 1

Total Number of Image Captures and Total Number of Target Captures for Each sUA

\begin{tabular}{|c|c|c|c|c|c|c|c|c|}
\hline \multirow[b]{2}{*}{ sUA } & \multicolumn{2}{|c|}{100 feet $\mathrm{AGL}$} & \multicolumn{2}{|c|}{200 feet $A G L$} & \multicolumn{2}{|c|}{300 feet $\mathrm{AGL}$} & \multicolumn{2}{|c|}{400 feet $\mathrm{AGL}$} \\
\hline & $\begin{array}{c}\text { Total } \\
\text { Images }\end{array}$ & $\begin{array}{c}\text { With } \\
\text { Target }\end{array}$ & $\begin{array}{c}\text { Total } \\
\text { Images }\end{array}$ & $\begin{array}{l}\text { With } \\
\text { Target }\end{array}$ & $\begin{array}{c}\text { Total } \\
\text { Images }\end{array}$ & $\begin{array}{c}\text { With } \\
\text { Target }\end{array}$ & $\begin{array}{c}\text { Total } \\
\text { Images }\end{array}$ & $\begin{array}{c}\text { With } \\
\text { Target }\end{array}$ \\
\hline Mavic 2 & 189 & 55 & 87 & 56 & 70 & 59 & 55 & 50 \\
\hline Bebop 2 & 110 & 55 & 72 & 46 & 72 & 46 & 75 & 46 \\
\hline Disco & 183 & 34 & 209 & 60 & 247 & 79 & 249 & 87 \\
\hline
\end{tabular}

Note. $\mathrm{sUA}=$ small unmanned aircraft; $\mathrm{AGL}=$ above ground level. 
The Pix4D application commands the sUA to capture aerial images at certain points in the flight plan and ensures that the sUA does not capture any images during transitions between each leg of the grid pattern, as shown in Figure 7.

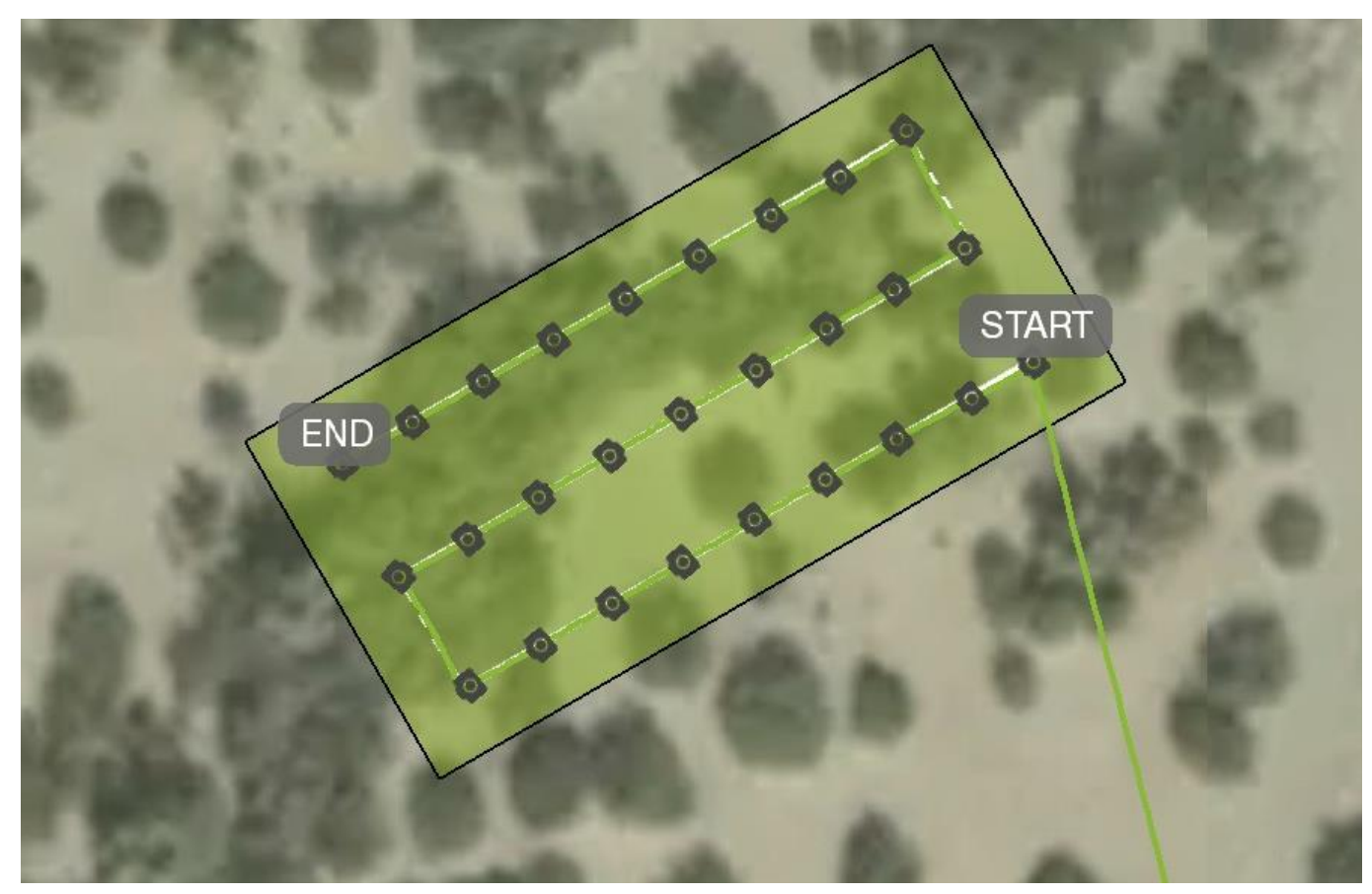

Figure 7. Screen capture of completed Mavic 2 Pro flight plan. The camera symbols indicate the location and the direction of the sensor when each image was taken.

The Parrot Disco used the FreeFlight Pro application on an iOS device (iPhone X or iPad Mini 5) for flight planning. The Disco was outfitted with a GoPro Hero8 camera which was not controlled by the flight planning software. The GoPro Hero8 captured images at a set time interval ( 2 seconds), regardless of if the sUA was over the target area or transitioning to the next leg. Additionally, the Parrot Disco is a fixed wing sUA design, and therefore cannot transition between flight legs in the same manner as the two quadcopter sUA and must instead make wide turns to transition between legs. The flight characteristics combined with the different method of image capture resulted in more image captures for the Parrot Disco. Additionally, as the image captures for the Disco are on a timed interval, rather than commanded, the number of target 
images captured increases with altitude for this sUA, as the target is within the camera's field of view for longer at higher altitudes. The overall counts for detection of each color garment are shown in Figure 8.

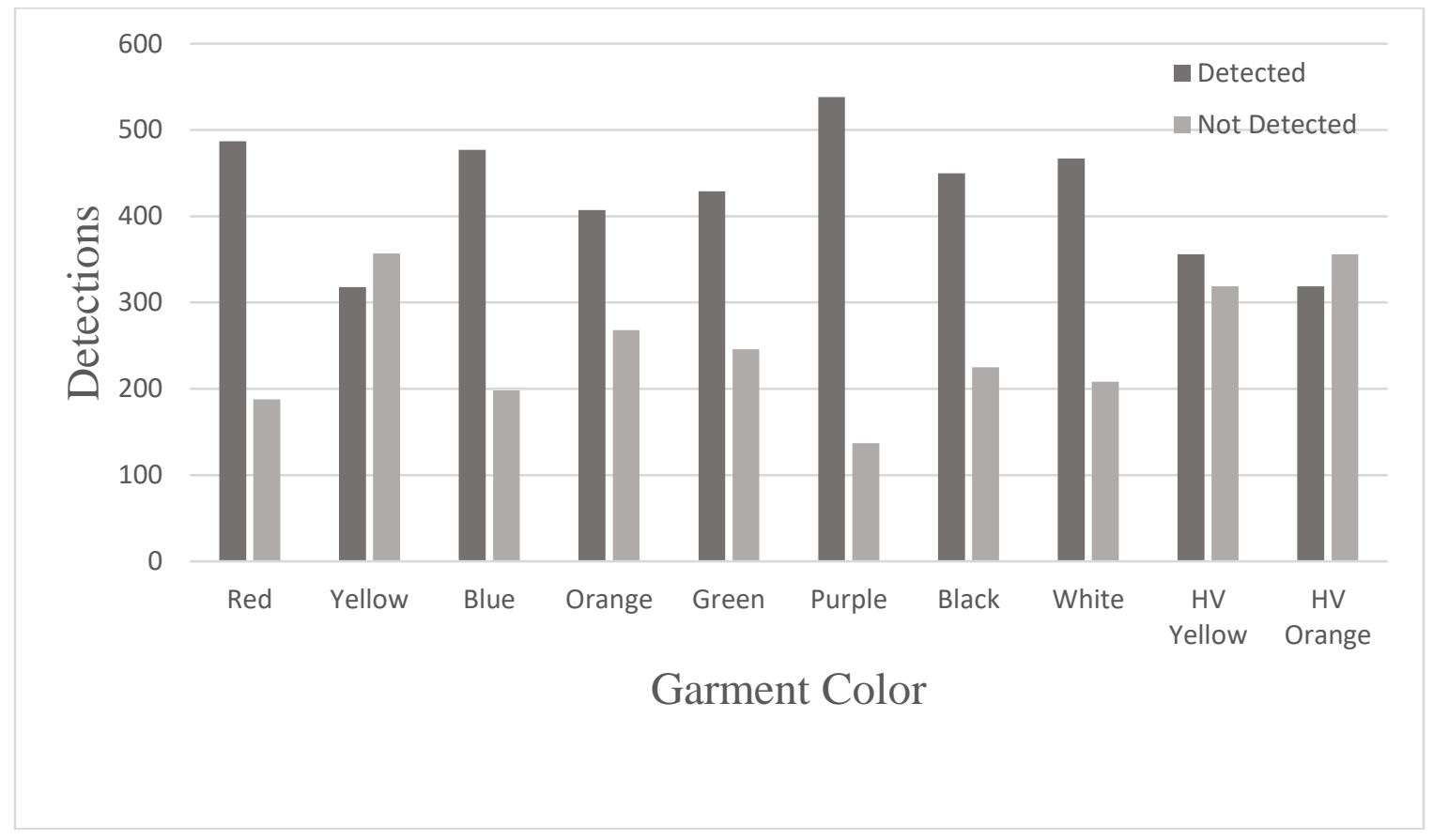

Figure 8. Overall successful detection versus missed detection counts by garment color. Each garment color had 72 total analyses conducted, for a total of 720 detection opportunities. Each sUA captured 18 total image sets for analysis. (HV = High Visibility).

For false positive detection rates, a standard was selected to provide a basis for designating a color as producing a high number of false positive detections compared to another color. For the purpose of this study, a high rate of false positive detections was defined as any individual analysis (single sUA at a single altitude) that returned over twice as many false positive results as successful detections in Loc $8^{\mathrm{TM}}$. For garment colors with high false positive detection rates, there were several instances where a single analysis produced over 100 false positive detections. For example, one mission from the Mavic 2 Pro at 200 feet AGL captured 24 aerial images. Out of the 24 images, 12 contained the target garment color, white. Loc $8^{\mathrm{TM}}$ flagged 23 out of the 24 images when commanded to search using the color profile built for the 
white garment. Operator analysis of the flagged images showed that Loc $8^{\mathrm{TM}}$ had only identified the white garment six times and flagged 212 other items within the images as potential matches. In the case of the colors black and purple, over half of all analyses resulted in over 100 false positive detections, as shown in Figure 9.

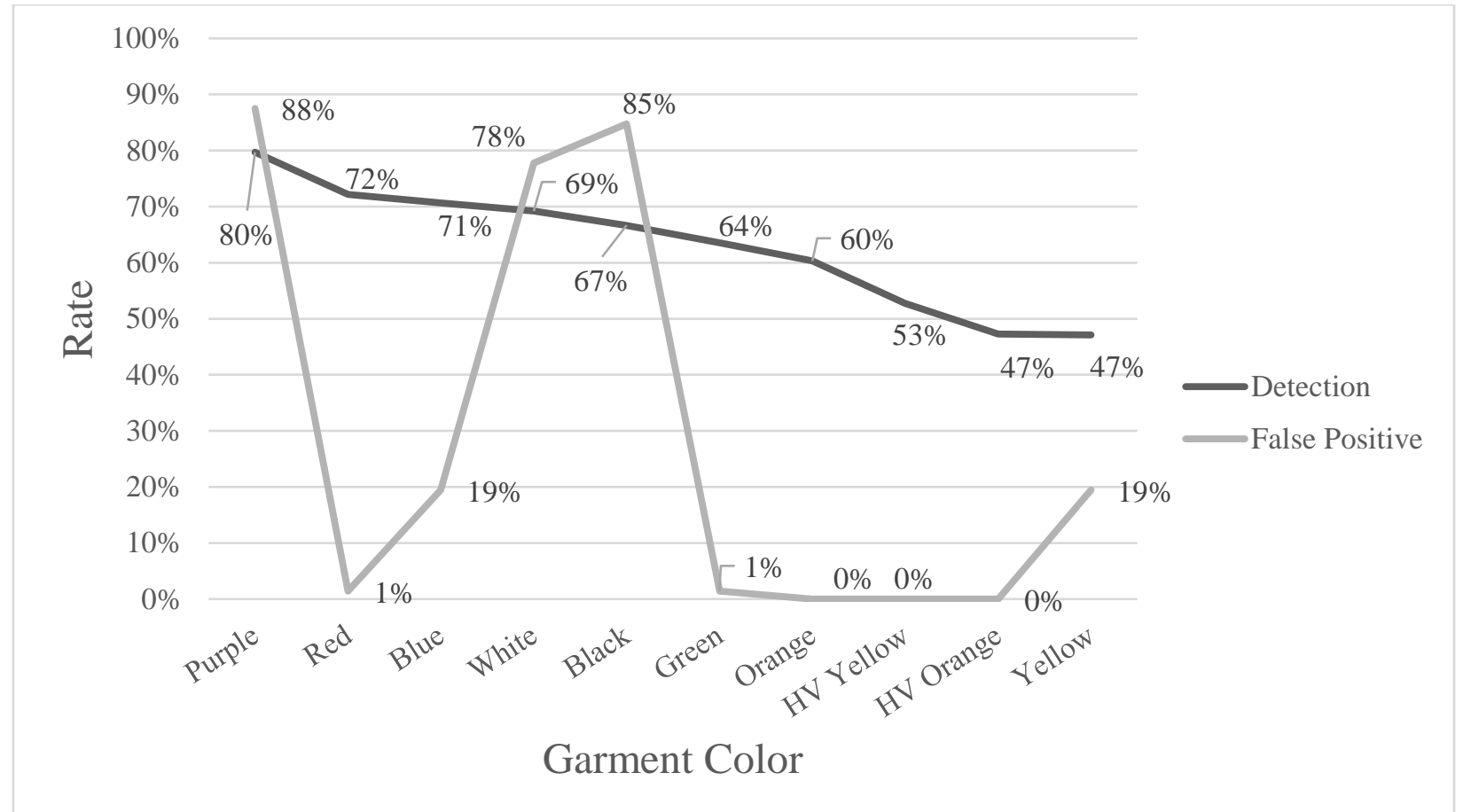

Figure 9. False positive detection rates significantly affected three garment colors. Two additional garment colors had minor false positive detection rates, with the remaining colors showing few to no significant false positive detections. In $80 \%$ of all analyses for the black garment with a high false positive detection rate, Loc ${ }^{\mathrm{TM}}$ returned over 100 flagged items as potential matches. The purple garment false positive rate includes $65 \%$ of analyses that returned over 100 flagged images. The white garment returned over 100 false positive detections in 59\% of the analyses with high false positive detections.

The mean successful detection rate for all garment colors was 63\% (SD = 10.5\%).

Detection rates for all colors varied based on the sUA used to collect the aerial images. The detection rate for aerial images captured by the Mavic 2 Pro was $86 \%$, the Parrot Disco was $62 \%$, and the Parrot Bebop 2 was 38\%. Detection rates also varied by image capture altitude. 
Flight altitude had an inverse relationship with detection rate, with an increase in flight altitude resulting in a decrease in detection rate, as shown in Figure 10. Overall detection rates were higher in the earlier parts of the day (morning and noon) and fell during the later parts of the day (afternoon and evening), as shown in Figure 11.

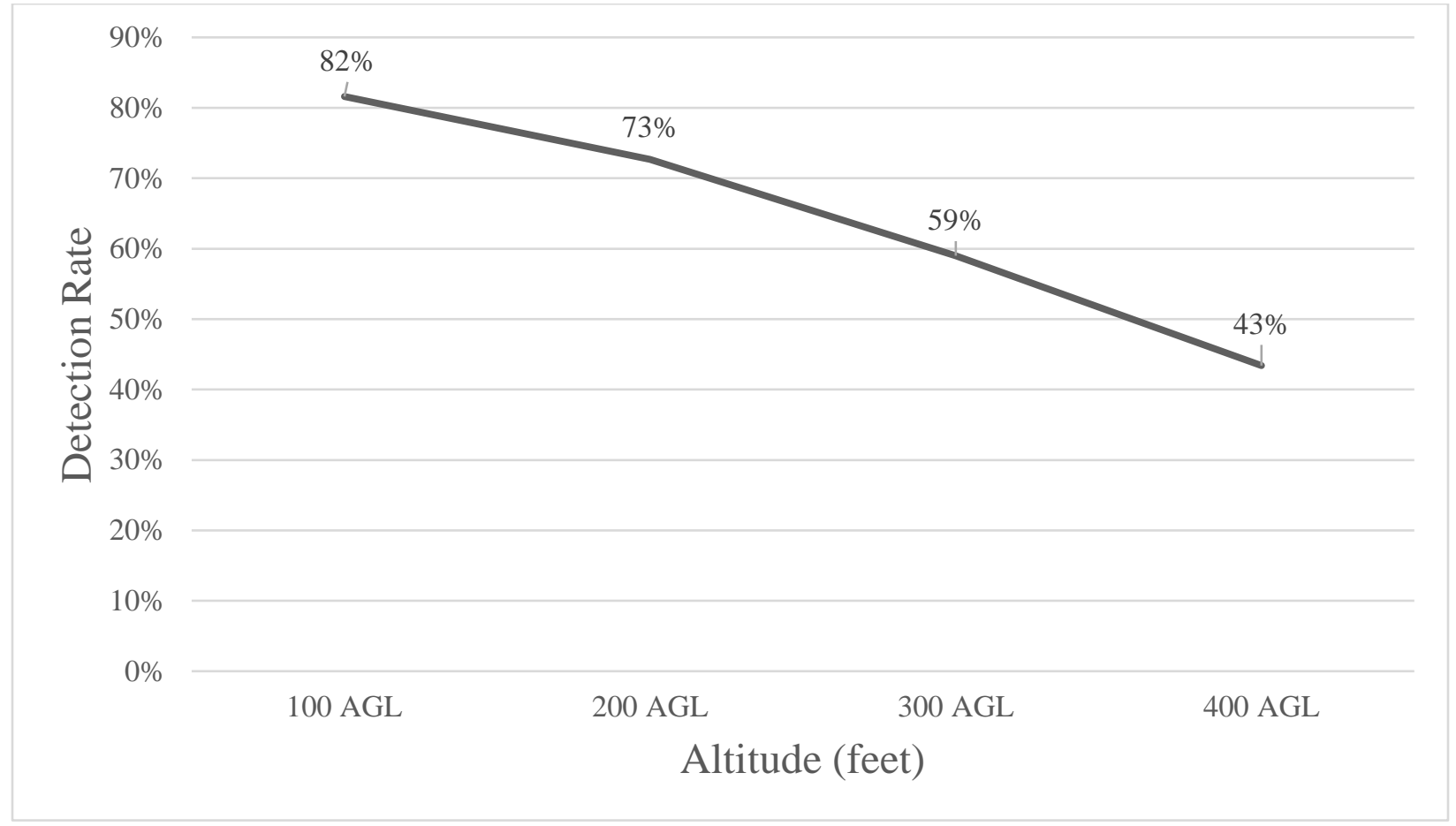

Figure 10. Overall detection rates per altitude flown. 


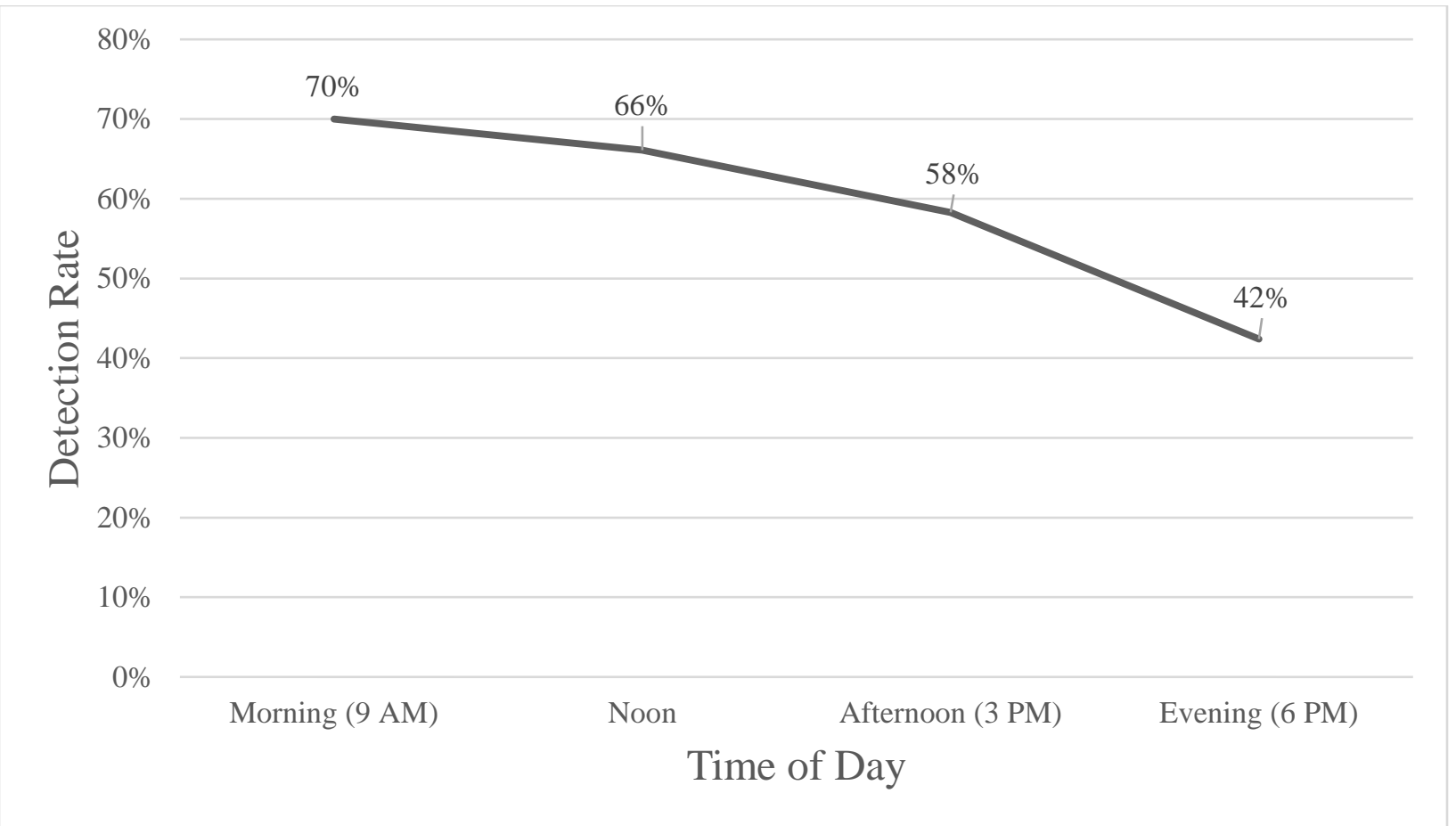

Figure 11. Detection rates by time of day.

Results of the imagery analysis were tabulated, and a chi-square test for independence was performed to determine if there was a relationship between garment color and detection rate. The relationship between these variables was significant, $\chi 2(9, \mathrm{~N}=6750)=314.31, \mathrm{p}<.001$. The p-value indicates that there is a statistically significant relationship between garment color and detection rate when analyzing aerial images from 12-20-megapixel cameras between 100400 feet AGL. The effect size was $\mathrm{V}=0.22$, which indicates a moderate relationship.

Separate chi-square tests for independence were performed on the individual sUA detected data. When tested individually the DJI Mavic 2 Pro $\chi^{2}(9, \mathrm{~N}=2200)=227.70, \mathrm{p}<$ .001 , Parrot Disco $\chi^{2}(9, \mathrm{~N}=2620)=510.55, \mathrm{p}<.001$, and Parrot Bebop $2 \chi^{2}(9, \mathrm{~N}=1930)=$ $317.09, \mathrm{p}<.001$ had statistically significant results. The relationship was stronger amongst the individual sUA (Mavic 2 Pro $\mathrm{V}=0.32$, Disco $\mathrm{V}=0.44$, Bebop $2 \mathrm{~V}=0.41$ ), with all three sUA indicating a strong relationship between garment color and detection rate. 


\section{Summary}

The mean successful detection rate for all garment colors was $63 \%(\mathrm{SD}=10.5)$.

Detection rates for all colors varied based on the sUA/sensor used to collect the aerial images.

The detection rate for aerial images captured were (a) $86 \%$ for the Mavic 2 Pro, (b) $62 \%$ for the Parrot Disco, and (c) $38 \%$ for the Parrot Bebop 2. Detection rates also varied by image capture altitude. Flight altitude had an inverse relationship with detection rate: (a) $82 \%$ detection rate at $100 \mathrm{ft} \mathrm{AGL;} \mathrm{(b)} 73 \%$ at $200 \mathrm{ft}$ AGL; (c) 59\% at $300 \mathrm{ft}$ AGL; and (d) $43 \%$ at $400 \mathrm{ft}$ AGL.

Overall detection rates were higher in the earlier parts of the day (morning and noon) and fell during the later parts of the day (afternoon and evening): (a) 70\% detection rate at 9 a.m.; (b) $66 \%$ at 12 p.m.; (c) $58 \%$ at 3 p.m.; and (d) $42 \%$ at 6 p.m. Of the top five garment colors based on detection rates, three (purple, black, and white) showed high rates of false positive detection. Red (72\% detection rate) and blue (71\% detection rate) were the garment colors with the highest detection rate that did not also include a high false detection rate.

\section{Conclusions}

The null hypothesis that clothing color would not have any effect on the ability to detect the missing person using image analysis software was rejected based on the results of the chisquare test: clothing color did have an effect on the ability to detect a missing person using image analysis software. The garment color with the highest detection rate in this study was purple. Loc $8^{\mathrm{TM}}$ imagery analysis was able to locate the torso model with the purple shirt in $80 \%$ of analyses. Which was $8 \%$ more frequently than the next highest color (red). High visibility orange and yellow showed the lowest rates of detection at only $47 \%$. The higher successful detection rate was not without detractors in some cases. While the purple garment was detected the most often, it also had the highest rate of false positive detections. The higher successful 
detection rate was not without detractors in some cases. While the purple garment was detected the most often, it also had the highest rate of false positive detections. False positive detections, as shown in Figure 12, were indicated as image flags by the Loc8 ${ }^{\mathrm{TM}}$ software, which would have to be manually analyzed by the SAR team to confirm a positive detection. Confirming dozens to hundreds of images that contain false positive detections is a time-consuming task that diminishes the benefits of using a sUAS aid to the search effort.

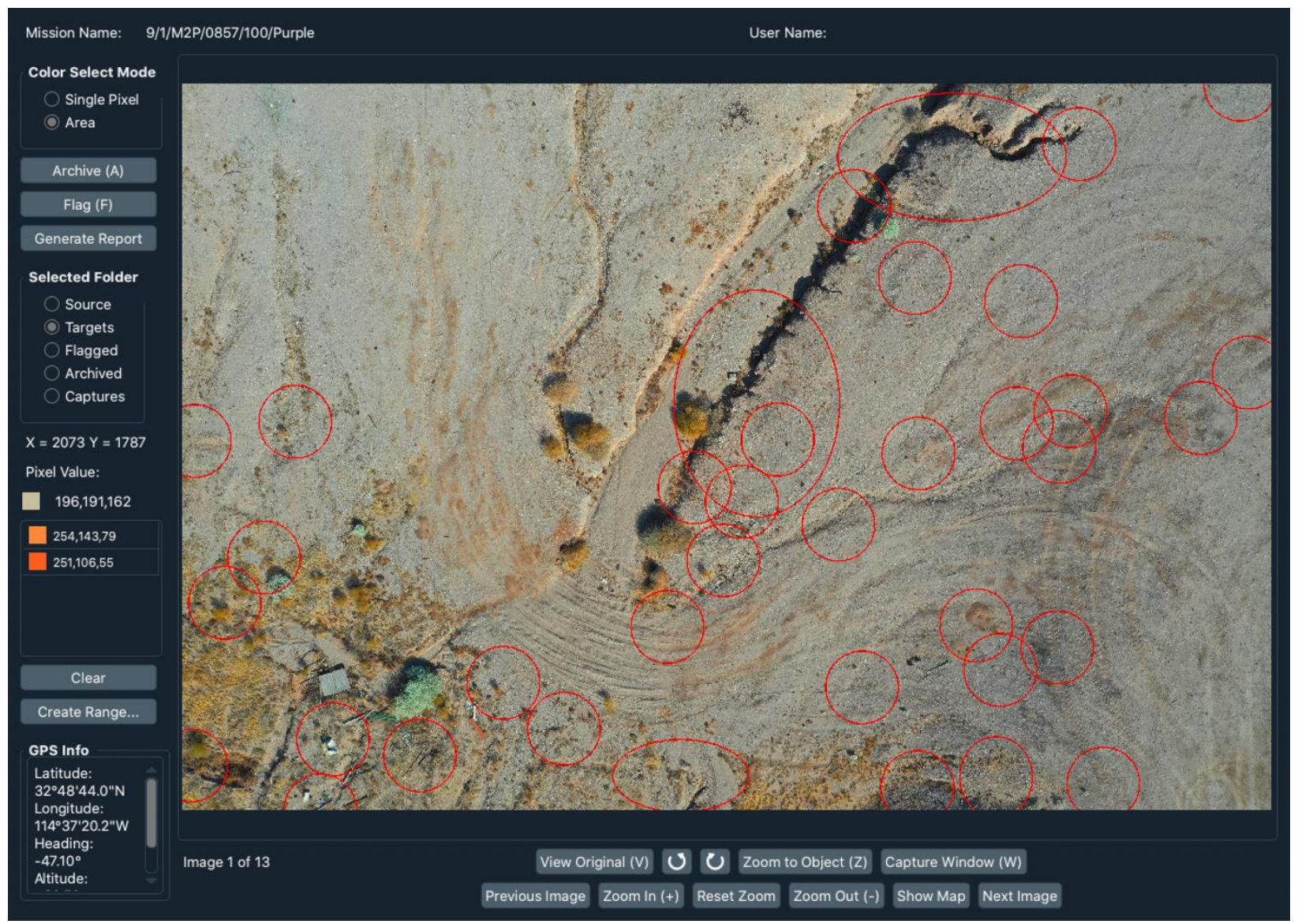

Figure 12. Screen capture of Loc $8^{\mathrm{TM}}$ program post analysis image flag for the color purple at 100 feet AGL. This image was captured by the Mavic 2 Pro; however, similar images were captured by all sUA during this research. The red circles in the image indicate pixels identified by Loc $8^{\mathrm{TM}}$ as matching the color profile selected by the operator for detection. In this image there are 37 areas circled as possible matches. Post search confirmation by the operator showed that all 37 instances were false positive detections, and the target was not captured in this particular image. 
In some instances, Loc8 ${ }^{\mathrm{TM}}$ flagged an image with several false detections and failed to flag the target color, which was also in the image, as shown in Figure 13. For color profiles determined to have a high false positive rate, such as purple, there were numerous instances where Loc $8^{\mathrm{TM}}$ flagged over half of all of the images searched. In many of the flagged images, the correct target would be identified; however, there would also be 10-20 false positive detections in the same image which can distract the operator from the actual target. In a large SAR operation, there can be hundreds, or thousands of images captured by sUA camera sensor. If Loc $8^{\mathrm{TM}}$ returned half of the images with only false positives, it would degrade the ability of the SAR team to effectively clear an area in an expeditious manner, defeating the purpose of using the sUA. Of the top five garment colors based on detection rates, three (purple, black, and white) showed high rates of false positive detection. Red (72\% detection rate) and blue (71\% detection rate) were the garment colors with the highest detection rate that did not also include a high false detection rate. The false positive detection rate for blue was $19 \%$ and only $1 \%$ for red, as shown in Figure 9. 


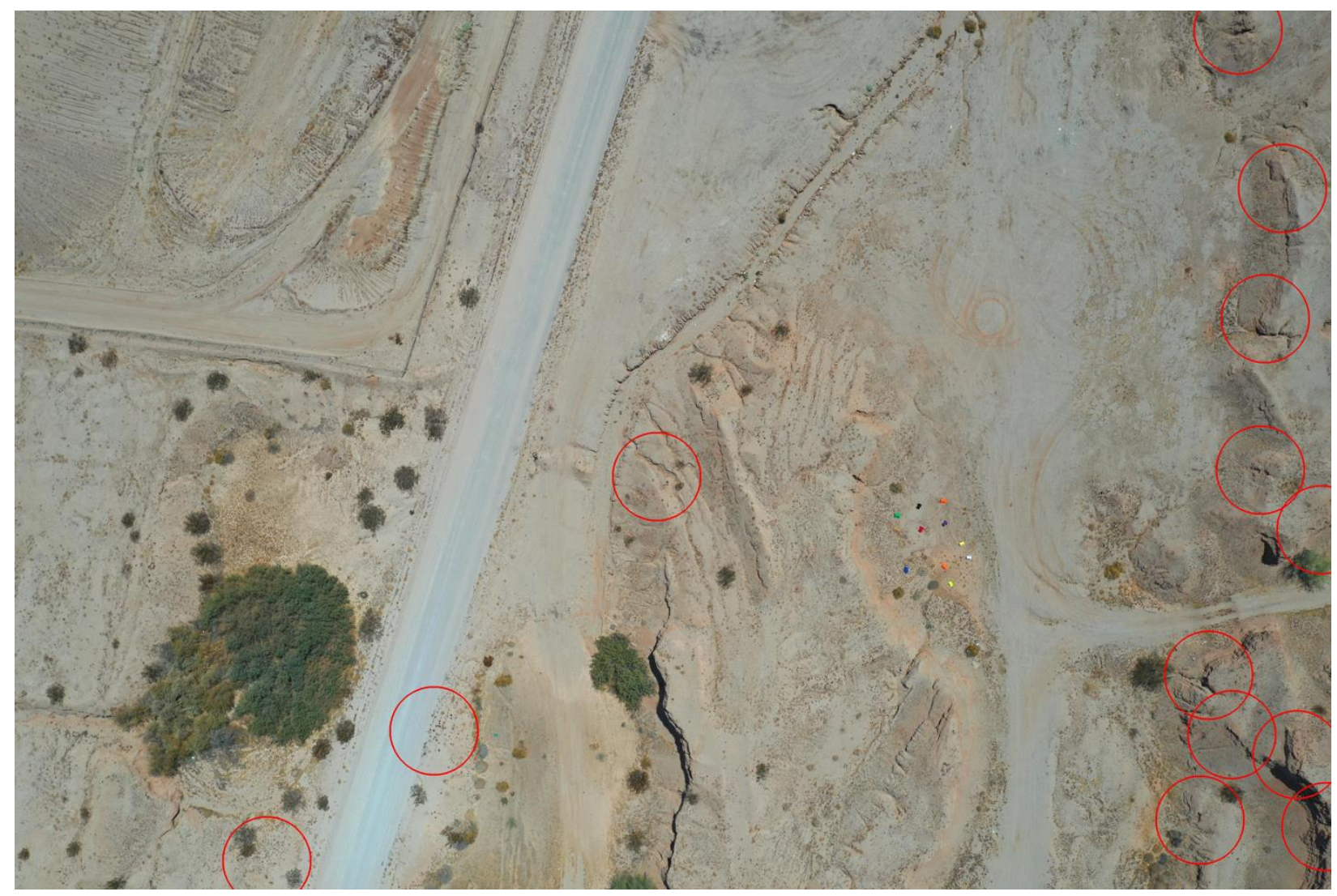

Figure 13. Flagged image captured by Mavic 2 Pro at 400 feet AGL. Target search color was purple. Loc $8^{\mathrm{TM}}$ identified 13 as possible matches. Operator analysis of the flagged image showed that all 13 areas were false positive detections. The torso model with the purple garment was also in the image; however, it was not flagged by Loc8 ${ }^{\mathrm{TM}}$ as a possible match.

The false detection rate for blue was elevated mainly due to the angle of the Bebop 2 camera, which caused images from that sUA to include the skyline as well as the surface, resulting in most of the false positives. Without the inclusion of the Bebop 2 images, the false detection rate for blue would fall to $3 \%$. The angle of the Bebop 2 camera did not have a significant effect on any other color garment. The significantly lower false detection rate of red makes it the superior color to purple for automated detection, as shown in Table 2. 
Table 2

Purple and Red Detection and False Positive Data Comparison

\begin{tabular}{cccccccc}
\hline $\begin{array}{c}\text { Garment } \\
\text { Color }\end{array}$ & $\begin{array}{c}\text { Total } \\
\text { Images }\end{array}$ & $\begin{array}{c}\text { Images } \\
\text { with } \\
\text { Target }\end{array}$ & $\begin{array}{c}\text { \% of } \\
\text { Images } \\
\text { with } \\
\text { Target }\end{array}$ & Detected & $\begin{array}{c}\text { Not } \\
\text { Detected }\end{array}$ & $\begin{array}{c}\text { Total } \\
\text { Flagged } \\
\text { Images }\end{array}$ & $\begin{array}{c}\% \text { of } \\
\text { Flagged } \\
\text { Images }\end{array}$ \\
\hline Purple & 1688 & 675 & $40 \%$ & 538 & 137 & 1291 & $76.5 \%$ \\
Red & 1688 & 675 & $40 \%$ & 487 & 188 & 789 & $46.7 \%$ \\
\hline
\end{tabular}

Note. In an actual SAR operation, the total number of images can remain high, with a significantly lower number of images with the target present.

Altitude was also a major contributing factor for detection using Loc8 ${ }^{\mathrm{TM}}$. As the altitude of the missions increased, the quality of the aerial images decreased, thus decreasing the detection rate, as shown in Figure 10. Altitude is an important factor in SAR operations; the higher the sUA is flown at, the more area that the camera can image in a single flight. For example, a one square mile search area, using a standard grid flight pattern in Pix4D Capture, would take the Mavic 2 Pro 24.11 hours to image at 100 feet AGL, not including time for battery changes. The same one square mile search area could be imaged by the Mavic 2 Pro in only 1.7 hours at 400 feet AGL. However, even the Mavic 2 Pro only produced a $77 \%$ successful detection rate at 400 feet AGL. To ensure a minimum 90\% success rate, the SAR team would have to limit the Mavic 2 Pro to 200 feet AGL (90\% detection rate) or use the Parrot Disco at 100 feet AGL (90\% detection rate). To provide the highest chance of success, the SAR team must know the limitations of the sUA they are employing based on the planned altitude of the flights.

The Mavic 2 Pro, with its 20-megapixel camera, was able to record aerial images that were superior in quality to the other two small unmanned aircraft (sUA). The larger images resulted in an overall detection rate of $86 \%$ for the Mavic 2 Pro. The Parrot Bebop 2 and the 
GoPro Hero8 camera used on the Parrot Disco produce 14 and 12-megapixel images, respectively. Aerial images from the Disco resulted in a detection rate of $62 \%$, which is significantly higher than the $38 \%$ detection rate observed with the Bebop 2 . This study demonstrated that the colors captured by image analysis vary substantially depending on the sensor specifications and mounting location (fixed or gimballed) on the sUA, as well as the altitude and the time of day.

The null hypothesis that clothing color would not have any effect on the software's ability to detect the missing person was rejected based on the results of the chi-square test; clothing color did have an effect on the software's ability to detect a missing person. The garment color with the highest detection rate in this study was purple. Loc8 ${ }^{\mathrm{TM}}$ imagery analysis was able to locate the torso model with the purple shirt in $80 \%$ of analyses, $8 \%$ more frequently than the next highest color, red. High visibility orange and yellow showed the lowest rates of detection at only $47 \%$. The higher successful detection rate was not without detractors in some cases. While the purple garment was detected the most often, it also had the highest rate of false positive detections. False positive detections were indicated as image flags by the Loc $8^{\mathrm{TM}}$ software, which must be further analyzed by the SAR team to confirm a positive detection, as shown in Figure 12. Confirming dozens to hundreds of images that only contain false positive detections is a time-consuming task that does not provide any aid to the search effort.

The selection of the small unmanned aircraft system (sUAS) played a major role in the success or failure of the Loc8 ${ }^{\mathrm{TM}}$ software to detect the selected garment color. The Mavic 2 Pro, with its 20-megapixel camera, was able to record aerial images that were superior in quality to the other two small unmanned aircraft (sUA). The larger images resulted in an overall detection rate of $86 \%$ for the Mavic 2 Pro. The Parrot Bebop 2 and the GoPro Hero 8 camera used on the 
Parrot Disco produce 14 and 12-megapixel images, respectively. The main difference in how the Bebop 2 and Disco captured images was the angle of the camera. The Disco captured nadir images, whereas the Bebop 2 captured images at a 30-degree angle, due to software limitations within the flight planning software. The angle of capture appears to have created a disadvantage for the Bebop 2, despite the larger pixel size of the captured images. Aerial images from the Disco resulted in a detection rate of $62 \%$, which is significantly higher than the $38 \%$ detection rate observed with the Bebop 2, as shown in Figure 14. When conducting SAR operations, the use of either of the Parrot sUA would have a high probability of mission failure. At the lowest altitude flown, 100 feet AGL, the images from the Bebop 2 resulted in a detection rate of $65 \%$, over 10\% lower than the detection rate of the Mavic 2 Pro at the highest altitude flown of 400 feet AGL (77\%). The Parrot Bebop 2's camera performed best when the terrain being searched was relatively flat with no vegetation or topography that could mask a potential victim or object of interest. Even with a grid pattern that allowed the Bebop 2 to capture images from two different directions, there were still several instances where foliage blocked the camera from capturing a clear image of the torso model, thus contributing to the poor overall performance of the Bebop 2 images in post analysis. 


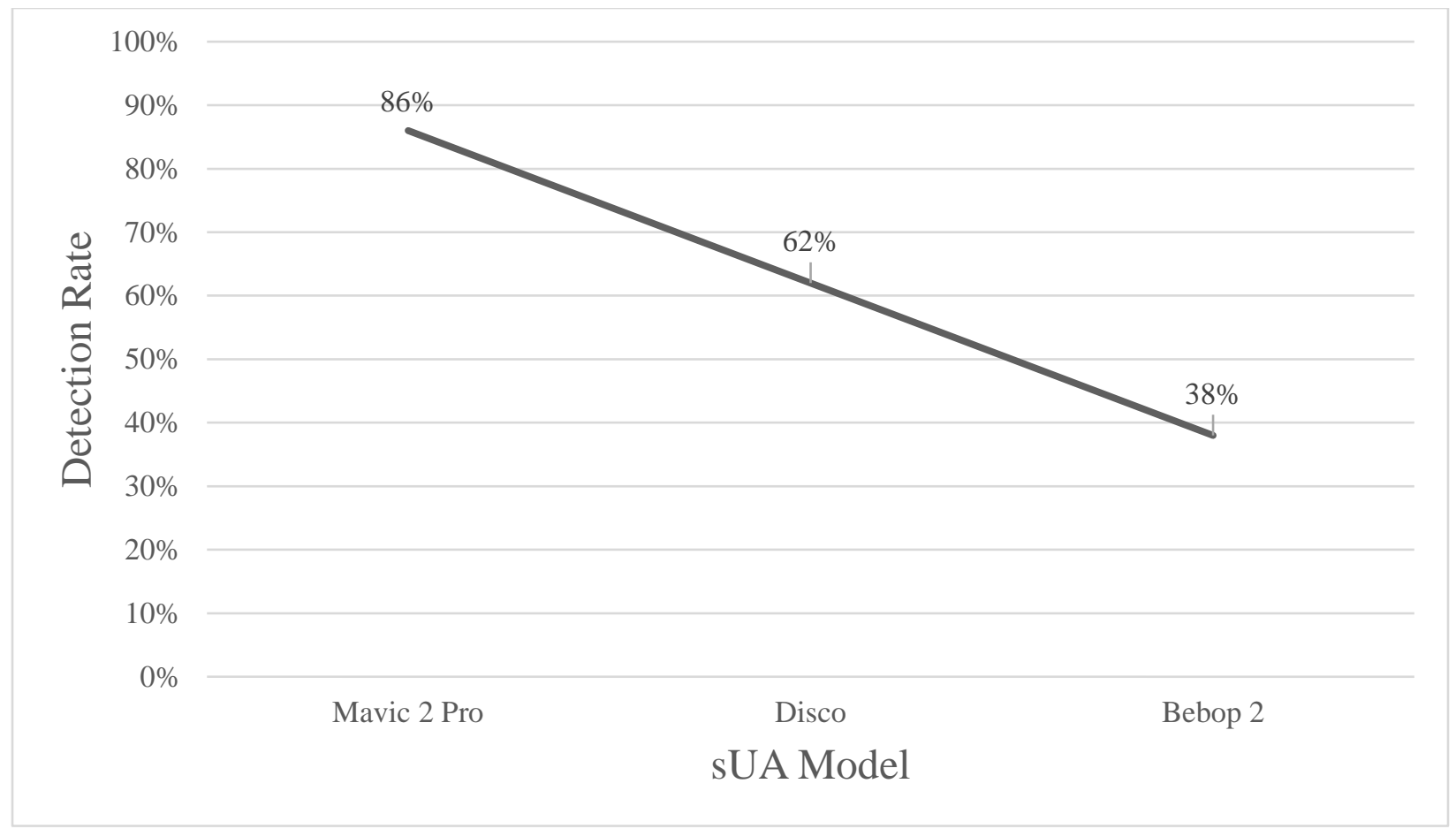

Figure 14. Overall detection rates per sUA.

Only limited data is available for flights later in the day (afternoon and evening) due to extremely high temperatures that exceeded the maximum ambient operating temperature ranges of the sUA. When comparing the limited data with data collected in the earlier parts of the day, there is an overall decrease in detectability as the day progresses, although some sUAS-specific detection rates fluctuated throughout the day. Flights conducted at 9 a.m. resulted in a detection rate of $70 \%$, whereas flights conducted at 6 p.m. had only a $42 \%$ detection rate, as shown in Figure 11. This was most prevalent with the two Parrot sUA. The Parrot Bebop 2 suffered from a progressive $18 \%$ decrease in detection rates between 9 a.m. and 3 p.m., with only a $3 \%$ detection rate by 6 p.m. The Parrot Disco detection rate decreased $30 \%$ between noon and 3 p.m., although the Disco detection rate did rise $19 \%$ between 3 p.m. and 6 p.m. The Mavic 2 Pro detection rates rose as the day progressed, with detection rates of $87 \%$ at 9 a.m., $88 \%$ at noon, and $94 \%$ at 3 p.m. However, the Mavic 2 Pro suffered a reduction of $23 \%$ in its detection rate by 
6 p.m. Part of this disparity between image captures at different times of the day is caused by the shift in the sun's angle and position, which changes the length and direction of shadows. All three sUA were flown in patterns that allowed image captures of the target area at two opposite angles (e.g. East to West, then West to East). Additionally, the angle of the sun can have an effect on the amount of light reflected off the garment, causing a shift in the color detected by the sUA (Smithson, 2005). Even a small shift could move the RGB values associated with a particular pixel far enough out of the range defined by the user that the Loc8 ${ }^{\mathrm{TM}}$ program will not flag them.

For this study, the Bloodhound Technique was used to create a color profile. In the event that a picture of the missing person is not available, a color profile can be built so long as the SAR team knows what color garments the person was wearing when last seen, although this method may be less effective due in large part to the many available shades of any given color that could be selected. One of the potential reasons for the decrease in detection accuracy later in the day found during this study may be that the image used to define the color profile for the Bloodhound Technique was taken earlier in the day. Teams using sUA and Loc8 ${ }^{\mathrm{TM}}$ (or similar programs) in SAR operations should be cognizant of the time of day the image of the missing person was taken and the time of day of the search. The color profile may need to be adjusted based on the different amount and angle of the light available to ensure detection rates remain high at opposite times of the day. For example, if the last known image of the missing person was taken at noon, the pixel color average that is obtained using the Bloodhound Technique in Loc $8^{\mathrm{TM}}$ may not be accurate for a search conducted near sunset. In this case, the color profile can be widened past the default range of 10 , or the RGB value for the average pixel color can be adjusted prior to selecting a range. 


\section{Recommendations}

This research was limited to rural/remote desert summer daytime environments only, however, SAR operations take place in other environment types as well. Follow-on research should be conducted to expand upon this study to explore the effectiveness of various garment colors in additional environment types. The colors that were found to be most effective for detection by Loc $8^{\mathrm{TM}}$ in the desert may not perform well in other areas. Colors with high contrast

(e.g. high visibility yellow and high visibility orange) to the surrounding environment were also not always as successful as expected, considering their recommendation as highly visible colors by organizations such as the IHEA and the Forest Service.

Additionally, sUAS platforms that use fixed angle sensors that do not allow the operator to capture nadir images, either because of limitations with the sUAS itself or the flight planning software, should be avoided. This would enable the sUA camera sensor to avoid capturing both the skyline and the ground in image captures, potentially causing additional false positive detections as was seen with blue when imaged by the Parrot Bebop 2 in this study.

The number of state and local police, sheriff, fire, and emergency services agencies in the U.S. that have acquired sUAS has continued to increase from less than 50 public service agencies in 2014 to over 1,500 as of March 2020 (Gettinger, 2020). Agencies and organizations that currently utilize sUAS for SAR operations, and those that intend to include them in their future capabilities, should conduct a small-scale study with their selected equipment since there could be significant variation in color detectability based on combinations of sUAS, optical sensor, and image analysis software. Having a predetermined profile for a variety of colors will aid the SAR team in future operations by allowing them to see how their chosen sensor captures colors of interest, so they can plan their imagery analysis appropriately. This study should be 
conducted with image captures from multiple altitudes to determine where the sUA performs best. To mitigate potential issues with color shifts at different times of the day, this study should collect aerial images of the same area and colors at multiple times throughout the day thereby providing a variety of atmospheric conditions, light levels, and shadowing/glare. 


\section{References}

Adams, A. L., Schmidt, T. A., Newgard, C. D., Federiuk, C. S., Christie, M., Scorvo, S., \& DeFreest, M. (2007). Search is a time-critical event: When search and rescue missions may become futile. Wilderness \& Environmental Medicine, 18(2), 95-101. https://doi.org/10.1580/06-WEME-OR-035R1.1

Agcayazi, M. T., Cawi, E., Jurgenson, A., Ghassemi, P., \& Cook, G. (2016, June). ResQuad: Toward a semi-autonomous wilderness search and rescue unmanned aerial system. Paper presented at the 2016 International Conference on Unmanned Aircraft Systems, Arlington, VA, USA, 898-904. https://doi.org/10.1109/ICUAS.2016.7502618

Al-Kaff, A., Gómez-Silva, M., Moreno, F., Escalera, A., \& Armingol, J. (2019). An appearancebased tracking algorithm for aerial search and rescue purposes. Sensors, 19(3), 652. https://doi.org/10.3390/s19030652

Alfio, V. S., Costantino, D., \& Pepe, M. (2020). Influence of image TIFF format and JPEG compression level in the accuracy of the $3 \mathrm{D}$ model and quality of the orthophoto in UAV photogrammetry. Journal of Imaging, 6(5), 30. https://doi.org/10.3390/jimaging6050030

Antunes, J. (2020). Loc8 locates people and objects quickly and effectively. Retrieved from https://www.commercialuavnews.com/public-safety/loc8-locates-people-and-objectsquickly-and-effectively

Boyles, J. (2017). Amazing aerial photography with Parrot Disco fixed wing drone and Bebop quadcopter. Retrieved from https://gigadgetstech.com/amazing-aerial-photographyparrot-disco-fixed-wing-drone-bebop-quadcopter/ 
Christie, K. S., Gilbert, S. L., Brown, C. L., Hatfield, M., \& Hanson, L. (2016). Unmanned aircraft systems in wildlife research: Current and future applications of a transformative technology. Frontiers in Ecology and the Environment, 14(5), 241-251. doi:10.1002/ fee. 1281

Cooper, J., \& Goodrich, M. (2008, March). Towards combining UAV and sensor operator roles in UAV-enabled visual search. Paper presented at the 3rd ACM/IEEE International Conference on Human Robot Interaction, Amsterdam The Netherlands, 351-358. https://doi.org/10.1145/1349822.1349868

Croft, J. L., Pittman, D. J., \& Scialfa, C. T. (2007). Gaze behavior of spotters during an air-toground search. Human Factors: The Journal of the Human Factors and Ergonomics Society, 49(4), 671-678. https://doi.org/10.1518/001872007X215746

Dinh, T. D., Pirmagomedov, R., Pham, V. D., Ahmed, A. A., Kirichek, R., Glushakov, R., \& Vladyko, A. (2019). Unmanned aerial system-assisted wilderness search and rescue mission. International Journal of Distributed Sensor Networks, 15(6). https://doi.org/10.1177/1550147719850719

DJI. (n.d.). Drone rescues around the world. Retrieved from https://enterprise.dji.com/dronerescue-map/\#map1599279547675

Eyerman, J., Crispino, G., Zamarro, A., \& Durscher, R. (2018). Drone efficacy study: Evaluating the impact of drones for locating lost persons in search and rescue events. Retrieved from https://eena.org/?s=drone+efficacy+study 
Fernández-Lozano, J., González-Díez, A., Gutiérrez-Alonso, G., Carrasco, R., Pedraza, J., García-Talegón, J., . . Morellón, M. (2018). New perspectives for UAV-based modelling the Roman gold mining infrastructure in NW Spain. Minerals, 8(11), 518. https://doi.org/10.3390/min8110518

Gettinger, D. (2020). Public Safety Drones, (3rd ed.). Retrieved from https://dronecenter.bard.edu/files/2020/04/CSD-Public-Safety-Drones-3rd-edition.pdf

Goldman, J. (2016). Parrot Bebop drone 2 review. Retrieved from https://www.cnet.com/ reviews/parrot-bebop-drone-2-review/2/

Goodrich, M. A., Morse, B. S., Gerhardt, D., Cooper, J. L., Quigley, M., Adams, J. A., \& Humphrey, C. (2008). Supporting wilderness search and rescue using a camera-equipped mini UAV. Journal of Field Robotics, 25(1-2), 89-110. https://doi.org/10.1002/rob.20226

GoPro. (n.d.). Hero8 Black manual. Retrieved from https://gopro.com/content/dam/help/hero8black/manuals/HERO8Black_UM_ENG_REVB.pdf

Grissom, C. K., Thomas, F., \& James, B. (2006). Medical helicopters in wilderness search and rescue operations. Air Medical Journal, 25(1), 18-25. https://doi.org/10.1016/j.amj.2005.10.002

Heggie, T. W., \& Amundson, M. E. (2009). Dead men walking: Search and rescue in U.S. national parks. Wilderness \& Environmental Medicine, 20(3), 244-249. doi:10.1580/08WEME-OR-299R.1

Heid, M. (2010). The brightest, most visible safety colors for cyclists and runners. Retrieved from https://www.outdoors.org/articles/amc-outdoors/the-brightest-most-visible-safetycolors-for-cyclists-and-runners 
Hoai, D. K., \& Phuong, N. V. (2017, October). Anomaly color detection on UAV images for search and rescue works. Paper presented at the Ninth International Conference on Knowledge and Systems Engineering (KSE), Hue, Vietnam. https://doi.org/10.1109/KSE.2017.8119473

Hunold, K. (1999). Digital videotapes formats. Broadcast Engineering, 41(8), 80-82. Retrieved from https://search-proquest-com.ezproxy.libproxy.db.erau.edu/docview/204161925/ abstract/689B495999434AAFPQ/1?accountid=27203

International Hunter Education Association (IHEA). (n.d.). Hunter orange requirements. Retrieved from http://www.ihea-usa.org/hunting-and-shooting/requirements/hunterorange-requirements

Khan, B. N. (2015). Hierarchical, low-cost person detection system for rescue and relief (Master's thesis). Retrieved from ProQuest One Academic database. (1781241161)

Loc8. (n.d.-a). Image scanning software. Retrieved from https://loc8.life

Loc8. (n.d.-b). Knowledge center: Bloodhound technique. Retrieved from https://help.loc8.life/ category/31-bloodhound-technique

Marshall, T., \& Perkins, N. (2015). Color outlier detection for search and rescue (Technical Report No. ECE-2015-01). Retrieved from https://pdfs.semanticscholar.org/0a58/cf003 1cad4f22391dbafec70b2bef653ceef.pdf

Michael. (2020, August 19). DJI Osmo Action vs GoPro Hero8 Black [Web blog post]. Retrieved from https://actiongadgetsreviews.com/dji-osmo-action-vs-gopro-hero8-black 
Morse, B., Thornton, D., \& Goodrich, M. (2012). Color anomaly detection and suggestion for wilderness search and rescue. Proceedings of the Seventh Annual ACM/IEEE International Conference on Human-Robot Interaction, 455-462. doi:10.1145/ 2157689.2157837

National Institute for Aviation Research. (2013). Hybrid II and Federal Aviation Administration Hybrid III anthropomorphic test dummy dynamic evaluation test series (Report No. DOT/FAA/AR-11/24). Retrieved from http://www.tc.faa.gov/its/worldpac/techrpt/ar1124.pdf

National Park Service (NPS). (2020). 2017 annual SAR dashboard. Retrieved from https://nps.maps.arcgis.com/apps/opsdashboard/index.html\#/b526c87ae21f4a669eb6c923 $8 \mathrm{c} 2 \mathrm{c} 4 \mathrm{bcf}$

Nicolas. (2015, August 24). Re: Focal length of camera [Online forum comment]. Retrieved from https://forum.developer.parrot.com/t/focal-length-of-camera/311

Pagliari, D., \& Pinto, L. (2018). Use of fisheye Parrot Bebop 2 images for 3d modelling using commercial photogrammetric software. International Archives of the Photogrammetry, Remote Sensing and Spatial Information Sciences, XLII-2, 813-820. https://doi.org/10.5194/isprs-archives-XLII-2-813-2018

Parrot. (2019). Flight plan. Retrieved from https://support.parrot.com/eu/support/products/parrotdisco-fpv/flight-plan

Pix4D. (2018). Pix4Dcapture - manual and settings. Retrieved from https://support.pix4d.com/ hc/en-us/articles/204010419-iOS-Pix4Dcapture-Manual-and-Settings

Pix4D. (n.d.). Supported drones, cameras and controllers. Retrieved from https://support.pix4d .com/hc/en-us/articles/203991609-Supported-drones-cameras-and-controllers 
Robinson, G. (2012). First to deploy: Unmanned aircraft for SAR \& law enforcement. Retrieved from https://www.dropbox.com/s/dl/owqyvkaj1fdvt0s/First\%20To\%20Deploy\%20\%20Unmanned\%20Aircraft\%20for\%20SAR\%20and\%20Law\%20Enforcement.pdf

Skyvue 3D (Producer). (2017). Disco hero5 modification [YouTube]. Available from https:// www.youtube.com/watch?v=DwRdzzFl6J4

Smithson, H. E. (2005). Sensory, computational and cognitive components of human colour constancy. Philosophical Transactions of the Royal Society B, 360(1458). 1329-1346. https://doi.org/10.1098/rstb.2005.1633

Suciu, G., Dragu, M., Hussain, I., Iliescu, A., Orza, O., \& Mocanu, C. (2018, October). 3D modeling using Parrot Bebop 2 FPV. Paper presented at the 16th International Conference on Embedded and Ubiquitous Computing (EUC), Bucharest, Romania, pp. 61-65. https://doi.org/10.1109/EUC.2018.00016

Sun, J., Li, B., Jiang, Y., \& Wen, C.-Y. (2016). A camera-based target detection and positioning UAV system for search and rescue (SAR) purposes. Sensors, 16(11), 1778. https://doi.org/10.3390/s16111778

3M. (2016). Standard update \#34: ANSI/ISEA 107-2015 made easier. Retrieved from https:// multimedia.3m.com/mws/media/638577O/ansi-107-2015-made-easier-high-visibilityapparel.pdf

United States Coast Guard (USCG). (2019). United States Coast Guard search and rescue summary statistics 1964 thru 2017. Retrieved from https://catalog.data.gov/dataset/uscgsearch-and-rescue-summary-statistics 
United States Forest Service. (n.d.). Hunting safety: Information for hunters and non-hunters. Retrieved from https://www.fs.usda.gov/Internet/FSE_DOCUMENTS/stelprdb533 0495.pdf

Van Tilburg, C. (2017). First report of using portable unmanned aircraft systems (drones) for search and rescue. Wilderness \& Environmental Medicine, 28(2), 116-118. https://doi.org/10.1016/j.wem.2016.12.010

Wang, G., Fu, R., Sun, B., Lv, J., Sheng, T., \& Tan, Y. (2017, November). Comparison of two types of color transfer algorithms in YUV and lab color spaces. Paper presented at the Applied Optics and Photonics Event, Beijing, China. doi:10.1117/12.2284953

Weldon, W., \& Hupy, J. (2020). Investigating methods for integrating unmanned aerial systems in search and rescue operations. Drones, 4(3), 38. https://doi.org/10.3390/drones4030038

Worley, G. H. (2015). Civilian helicopter search and rescue accidents in the United States: 1980 through 2013. Wilderness \& Environmental Medicine, 26(4), 544-548. https://doi.org/10.1016/j.wem.2015.08.001

Zaidi, F. H., Hull, J. T., Peirson, S., Wulff, K., Aeschbach, D., Gooley, J. J., . . Lockley, S. W. (2007). Short-wavelength light sensitivity of circadian, pupillary, and visual awareness in humans lacking an outer retina. Current Biology, 17(24), 2122-2128. https://doi.org/10.1016/j.cub.2007.11.034 